\title{
INVARIANT MEASURE FOR RANDOM WALKS ON ERGODIC ENVIRONMENTS ON A STRIP
}

\author{
By Dmitry Dolgopyat and Ilya Goldsheid \\ University of Maryland, USA and Queen Mary University of London, GB
}

Environment viewed from the particle is a powerful method of analyzing random walks (RW) in random environment (RE). It is well known that in this setting the environment process is a Markov chain on the set of environments. We study the fundamental question of existence of the density of the invariant measure of this Markov chain with respect to the measure on the set of environments for RW on a strip. We first describe all positive sub-exponentially growing solutions of the corresponding invariant density equation in the deterministic setting and then derive necessary and sufficient conditions for the existence of the density when the environment is ergodic in both the transient and the recurrent regimes. We also provide applications of our analysis to the question of positive and null recurrence, the study of the Green functions and to random walks on orbits of a dynamical system.

\section{Introduction.}

1.1. Brief historic remarks and motivations. The approach to the study of the asymptotic behaviour of random walks in random environments (RWRE) known under the name environment viewed from the particle was initiated by S. Kozlov [21, 22], as well as by Papanicolau-Varadhan [26]. In the case of the RWRE in $\mathbb{Z}^{d}$, the idea is to move, after each jump of the particle, the environment and the particle in the direction opposite to the jump. The particle thus remains at its initial position but does "see" the same environments it would be seeing while performing the usual random walk.

The corresponding random process is a Markov chain (MC) on the space of environments. It turns out [22] that if the random environment is stationary and ergodic with respect to the standard shift and if this MC has an invariant measure which is absolutely continuous with respect to the measure on the space of environments then:

(a) the absolutely continuous measure is unique;

(b) this invariant measure and the measure on the space of environments are absolutely continuous with respect to each other;

MSC 2010 subject classifications: Primary 60K37; secondary 60J05, 82C44

Keywords and phrases: RWRE, random walks on a strip, invariant measure

imsart-aop ver. 2014/10/16 file: InvarMesRWRE.tex date: September 18, 2018 
(c) the stationary $\mathrm{MC}$ on the set of environments whose initial distribution is the said invariant measure is ergodic (see [22], [5], or [34] for a more comprehensive discussion and proofs of these properties).

The main model we consider in this work is the RWRE on a strip introduced in [3]. Our main goal is to obtain a complete classification of the solutions to the invariant density equation and to describe the necessary and sufficient conditions for the existence of an invariant density of the MC on the space of ergodic random environments on the strip in both the transient and the recurrent regimes. (Here and below we often use the expression "invariant density" instead of "the density of the invariant measure".)

In many papers concerned with the study of the RWRE, establishing the existence of the invariant density has been a crucial (if not the major) part of the work (see $[2,6,33,17,18,30,7,23,5,31,29,8]$ and references therein). As a fundamental problem of the theory of RWRE, this question is interesting and important in its own right. But our motivation is also due to two more sources.

One is Sinai's paper [33] where he considers a random walk on a torus generated by an irrational shift satisfying certain diophantine conditions. In Section 6 of [33], Sinai lists several open problems. We quote those of them which will be solved in this paper

"Let $T$ be a measure preserving automorphism acting on a measure space $(M, \mathcal{M}, \mu)$ and $p<1$ be a positive $\mu$-a.e. on $M$. Consider a Markov chain where a point $x \in M$ jumps to $T x$ with probability $p(x)$ and to $T^{-1} x$ with probability $1-p(x)$. Problem: does this MC have an invariant measure equivalent to $\mu$ ? We believe that in the case of $\mathrm{T}$ with strong mixing properties like Anosov transitive diffeomorphisms the answer is negative. Probably this case is connected with random walks in random environments (see [32]). It would be interesting to extend the results of this paper to Markov chains where a point can jump from $x$ to $T^{i} x,|i| \leq i_{0}$."

The first of these questions was, to a large extent, answered by Kaloshin and Sinai in [17] and [18]. As will be seen in Section 6, our results allow us to answer a question which includes as a particular case the second of the above questions and also to complement the results obtained in [17], [18].

The other motivation is due to our paper [11] where we came across the necessity to control the behavior of the variance of a recurrent RWRE on a strip in a bounded potential. The only way to do that in the recurrent case that we are aware of is via constructing the invariant density for the walk on the space of environments. In [11], this construction was carried out under the assumption that a particular function of the environment satisfies the homological equation (see (5.6) below). This prompts a natural question: is 
(5.6) also necessary? As will be shown below, the answer is positive.

Let us mention previous results related to our work. The existence of the invariant density in the ballistic regime (positive speed of escaping to infinity) was first proved in [30]. The corresponding formula for the density under the same conditions was found in [16]. RWRE with bounded jumps on $\mathbb{Z}$ were studied in [7] where the solutions to the invariant density equation for this model were described. The RW with bounded jumps on $\mathbb{Z}$ is a particular case of the RWRE on a strip and in this sense we recover here the results from [7]. The way in which the study of the RW on a line is reduced to the study of the RW on a strip was explained in [3], [4], [15].

1.2. Informal discussion of the main results and of the approach. The precise statements of our main results require some preparation and will be given later. Here, we discuss them informally. To do that we have to introduce some notations which will be discussed in detail in section 2 .

Let $(\Omega, \mathcal{F}, \mathbb{P})$ be a probability space with $\Omega$ being the space of environments and let $T$ an ergodic automorphism of $\Omega$ preserving measure $\mathbb{P}$. The invariant density equation can be viewed in two ways.

First, it is derived as a functional equation for the density function $\rho$ : $\Omega \mapsto \mathbb{R}^{m}$. It has the form

$$
\boldsymbol{\rho}(\omega)=\boldsymbol{\rho}(T \omega) Q(T \omega)+\boldsymbol{\rho}(\omega) R(\omega)+\boldsymbol{\rho}\left(T^{-1} \omega\right) P\left(T^{-1} \omega\right),
$$

where $\omega \in \Omega$ is an environment, $\rho$ is a row-vector, $P(\omega), Q(\omega), R(\omega)$ are $m \times m$ matrices with non-negative entries and such that their sum is a stochastic matrix: $(P(\omega)+Q(\omega)+R(\omega)) \mathbf{1}=\mathbf{1}$. (Throughout the paper $\mathbf{1} \in \mathbb{R}^{m}$ is a column vector whose all components are 1.)

Second, for a given $\omega$ we consider the restriction of $\boldsymbol{\rho}(\cdot)$ to the points of the trajectory $T^{n} \omega,-\infty<n<\infty$. Namely, we set

$$
\pi_{n}=\boldsymbol{\rho}\left(T^{n} \omega\right), \quad\left(P_{n}, Q_{n}, R_{n}\right)=\left(P\left(T^{n} \omega\right), Q\left(T^{n} \omega\right), R\left(T^{n} \omega\right)\right)
$$

and obtain from (1.1) the equation

$$
\pi_{n}=\pi_{n+1} Q_{n+1}+\pi_{n} R_{n}+\pi_{n-1} P_{n-1}, \quad-\infty<n<\infty .
$$

Since $\boldsymbol{\rho} \geq 0$, we are interested only in non-negative solutions. Next, it is easy to see (cf. inequality (2.12)) that $\pi_{n}$ may grow at most linearly in $n$. However, our technique allows us (at no additional cost) to classify subexponentially growing solutions and we shall do exactly that. We call such solutions tempered. The idea now is to describe all non-negative tempered solutions to (1.3) as functions of the sequence $\left(P_{n}, Q_{n}, R_{n}\right)$ and then to

imsart-aop ver. 2014/10/16 file: InvarMesRWRE.tex date: September 18, 2018 
extract from this description the information required for the control of (1.1). Accordingly, our analysis consists of two parts.

In the first part we view equation (1.3) as deterministic in the sense that we solve it for a fixed sequence $\left(P_{n}, Q_{n}, R_{n}\right)$. This sequence is supposed to satisfy ellipticity assumptions (2.6) but the second relation in (1.2) plays no role in the deterministic analysis. Our deterministic results are as follows.

- Equation (1.3) admits the first integral $\mathfrak{c}=\pi_{n} P_{n} \mathbf{1}-\pi_{n+1} Q_{n+1} \mathbf{1}$.

This fundamental property of equation (1.3) was not known before. Lemma 4.6 proves this statement and also contains another, more technical definition of $\mathfrak{c}$. Both definitions play a crucial role in the proofs of our main results. It is remarkable that the existence of $\mathfrak{c}$ does not need any positivity assumption on the solution.

- We describe all non-negative tempered solutions to (1.3) as explicit functions of certain auxiliary vectors and matrices which can be efficiently computed from the sequence $\left(P_{n}, Q_{n}, R_{n}\right)$.

Theorem 4.12 is the exact, extended, and quite technical version of this statement.

- Equation (1.3) has at most 3 linearly independent positive solutions.

This statement is not particularly intuitive since under mild non degeneracy assumptions the space of solutions to (1.3) is $2 m$-dimensional.

The second, probabilistic part of our analysis is mainly concerned with the existence of the invariant density. This time the stationarity in the form of the second relation in (1.2) plays a very important role. We still work with (1.3) - but with its stationary version. The results for (1.1) follow since $\boldsymbol{\rho}(\omega)=\pi_{0}(\omega)$. Under (2.6) and ergodicity of $T$, the following properties hold with probability 1.

- If a solution to (1.3) is a stationary process then the first integral $\mathfrak{c}$ considered as a function of $\omega$ is a constant (does not depend on $\omega$ ).

- The $R W$ on a strip is transient iff (1.3) has a non-negative tempered stationary solution with $\mathfrak{c} \neq 0$. This solution is unique and can be normalized to become the invariant density iff $\boldsymbol{\rho}(\omega) \equiv \pi_{0}(\omega)$ is integrable.

- The $R W$ is recurrent iff equation (1.3) has a unique non-negative tempered solution with $\mathfrak{c}=0$. This solution (properly normalized) is the invariant density iff the related homological equation is solvable.

REMARK 1.1. (a) The exact, extended, and more technical versions of these statements are contained in Lemmas 5.2, 5.3 and Theorems 5.4, 5.5.

imsart-aop ver. 2014/10/16 file: InvarMesRWRE.tex date: September 18, 2018 
(b) Note the contrast with the deterministic case: a tempered non-negative solution is always unique (up to a multiplication by a positive constant).

(c) A positive solution to (1.3) with $\mathfrak{c}=0$ always exists but it grows exponentially in the transient case.

(d) The results from [33] and [11], which use the solvability of the homological equation for constructing the invariant density, do now get an important final touch: in the absence of this condition the invariant density simply does not exist.

Checking the existence of solutions to the homological equation may not be a straightforward task. However, this was done for at least two important cases. Namely, if $\left(P_{n}, Q_{n}, R_{n}\right)$ is an i.i.d. sequence then the related homological equation is equivalent to a certain system of algebraic equations $([4,11])$. And if this sequence is generated by a diophantine quasi-periodic shift on a torus then it can be verified for a smooth enough generating function (as in $[1,33,11])$.

(e) The above results can also be viewed as a new criteria for the recurrence and transience of the walk. Previously, these were stated in [3] in terms of the sign of the Lyapunov exponent $\boldsymbol{\lambda}^{+}$(defined by (3.19)). The two criteria are of course equivalent $\operatorname{since} \operatorname{sign}(\mathfrak{c})=-\operatorname{sign}\left(\boldsymbol{\lambda}^{+}\right)$as we shall see later. We note that the interplay between $\mathfrak{c}$ and $\boldsymbol{\lambda}^{+}$is an important part of our analysis.

1.3. Some applications. We turn to a brief discussion of some applications of our main results.

In section 6.1 we provide necessary and sufficient conditions for positive recurrence of the walk on a strip. This result makes use of the fact that invariant measure of the walk on a strip (not on environments!) satisfies the deterministic version of (1.3) and the relevant solution is always the one with $\mathfrak{c}=0$.

In section 6.3 we consider the Green function (GF) at $-\infty$ for a RW which escapes to $+\infty$. By definition, the GF is the limit of the expected number of visits to a given site when the starting point of the walk tends to $-\infty$. We prove that this GF is the unique monotone increasing (see Definition 4.7) solution to $(1.3)$ with $\mathfrak{c}=1$. It plays an important role in the study of the limit theorems since the sites where it is large serve as traps. The traps for RWRE were studied in many papers starting from [20]. For the state of art picture in this area see $[9,10,14,27,28,34]$ and references therein. We also note that the Green function described above plays an important role in the recent work on local limit theorems for transient $\operatorname{RWRE}([2,11,24])$.

Sections 6.2 and 6.4 deal with application of the random version of (1.3).

imsart-aop ver. 2014/10/16 file: InvarMesRWRE.tex date: September 18, 2018 
In section 6.2 we discuss the generalization of Sinai's model mentioned above. Apart of the answer to Sinai's question, we also explain what happens in the so called non-symmetric case. In particular, we prove that if $T$ is a uniquely ergodic transformation of a compact metric space then the invariant density always exists in the non-symmetry case. Note that the ergodic shift on a torus considered in [33] is a particular case of this class of transformations.

Finally in section 6.4 we show that in the transient case the existence of non-zero velocity of the walk is equivalent to the existence of the invariant density. Moreover, we show that the non-zero velocity of the walk is $\mathfrak{c}$.

1.4. Organization of the paper. The RW on the strip is defined in section 2.1. In section 2.2 we explain the exact definition of the walk on the space of environments and state the invariant density equation for this walk in Lemma 2.3 (its derivation is given in Appendix A). Section 3 contains preparatory results: we recall several definitions and facts known from previous work and also state a new recurrence criteria for a RW in the deterministic setting. Exact statements and proofs of the main results concerned with the deterministic version of the invariant density equation are in Section 4 . In Section 5 we use these results to derive the necessary and sufficient conditions for the existence of the invariant density in the transient and in the recurrent case. In Section 6, we consider some applications of our main results; the structure and the content of this section has been explained above. Appendix B gives the proof of the recurrence criterion sated in Theorem 3.5. Apart of being used in the proofs, this criterion is important in its own right; we prove it in the appendix since it is outside the main topic of the present paper.

We would like to thank O. Zeitouni for a useful discussion related to the subject of this paper.

\section{The walks on the strip and on the space of environments.}

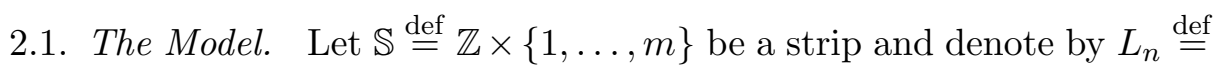
$\{(n, i): 1 \leq i \leq m\}$ the $n^{\text {th }}$ layer of the strip. The random walks on $\mathbb{S}$ with jumps to nearest layers were introduced in [3]: the walk is allowed to jump from any point $(n, i) \in L_{n}$ only to points in $L_{n-1}$, or $L_{n}$, or $L_{n+1}$. To describe the corresponding transition kernel consider a sequence of triples of $m \times m$ non-negative matrices, $\omega \stackrel{\text { def }}{=}\left(P_{n}, Q_{n}, R_{n}\right)_{-\infty<n<\infty}$, such that for all $n \in \mathbb{Z}$ the sum $P_{n}+Q_{n}+R_{n}$ is a stochastic matrix,

$$
\left(P_{n}+Q_{n}+R_{n}\right) \mathbf{1}=\mathbf{1}
$$

imsart-aop ver. 2014/10/16 file: InvarMesRWRE.tex date: September 18, 2018 
where $\mathbf{1}$ is a column vector whose components are all equal to 1 . The matrix elements of $P_{n}$ are denoted $P_{n}(i, j), 1 \leq i, j \leq m$, and similar notations are used for $Q_{n}$ and $R_{n}$. The transition kernel $\mathcal{Q}(\cdot, \cdot)$ is now defined as follows:

$$
\mathcal{Q}\left(z, z_{1}\right) \stackrel{\text { def }}{=} \begin{cases}P_{n}(i, j) & \text { if } z=(n, i), z_{1}=(n+1, j), \\ R_{n}(i, j) & \text { if } z=(n, i), z_{1}=(n, j), \\ Q_{n}(i, j) & \text { if } z=(n, i), z_{1}=(n-1, j), \\ 0 & \text { otherwise. }\end{cases}
$$

For a given $\omega$ and a starting point $z=(n, i) \in \mathbb{S}$ we define a random walk $\xi_{t}=\left(X_{t}, Y_{t}\right), t \in \mathbb{N}$, on $\mathbb{S}$ with transition kernel (2.2) in the usual way. Namely, the law $\mathcal{P}_{\omega, z}$ for the Markov chain $\xi$ with $\xi_{0}=z$ is given by

$$
\mathcal{P}_{\omega, z}\left(\xi_{1}=z_{1}, \ldots, \xi_{t}=z_{t}\right) \stackrel{\text { def }}{=} \mathcal{Q}_{\omega}\left(z, z_{1}\right) \mathcal{Q}_{\omega}\left(z_{1}, z_{2}\right) \cdots \mathcal{Q}_{\omega}\left(z_{t-1}, z_{t}\right) .
$$

We say that $\omega$ is the environment on the strip $\mathbb{S}$ and the walk $\xi$ is the walk in the environment $\omega$.

In this definition, $\omega$ is a fixed environment and, as mentioned before, some of our results are deterministic.

In the random setting we suppose that $(\Omega, \mathcal{F}, \mathbb{P}, T)$ is a dynamical system, where $\Omega$ is the space of all the sequences $\omega=\left(\left(P_{n}, Q_{n}, R_{n}\right)\right)_{n=-\infty}^{\infty}$ described above, $\mathcal{F}$ is the natural $\sigma$-algebra of subsets of $\Omega, \mathbb{P}$ denotes a probability measure on $(\Omega, \mathcal{F})$, and $T$ is the shift operator on $\Omega$ defined by $T\left(P_{n}, Q_{n}, R_{n}\right)=\left(P_{n+1}, Q_{n+1}, R_{n+1}\right)$ and preserving the measure $\mathbb{P}$. In this context we say that $\omega$ is a random environment on the strip $\mathbb{S}$.

Denote by $\Xi_{z}$ the set of trajectories $\xi_{t}, t \geq 0$ starting at $z . \mathcal{P}_{\omega, z}$ is the so called quenched probability measure on $\Xi_{z}$. The semi-direct product $\mathbb{P}(d \omega) \mathcal{P}_{\omega, z}(d \xi)$ of $\mathbb{P}$ and $\mathcal{P}_{\omega, z}$ is defined on the direct product $\Omega \times \Xi_{z}$ and is called the annealed measure. The corresponding expectations are denoted $\mathbb{E}, \mathrm{E}_{\omega, z}$, and $\mathbb{E}\left(\mathrm{E}_{\omega, z}(\cdot)\right)$ respectively.

Denote by $\mathcal{J}$ the following set of triples of $m \times m$ matrices:

$$
\mathcal{J} \stackrel{\text { def }}{=}\{(P, Q, R): P \geq 0, Q \geq 0, R \geq 0 \text { and }(P+Q+R) \mathbf{1}=\mathbf{1}\} .
$$

Let $\mathcal{J}_{0}=\mathcal{J}_{0}(\mathbb{P}) \subset \mathcal{J}$ be the support of the probability distribution of the random triple $\left(P_{n}, Q_{n}, R_{n}\right)$ defined above (obviously, this support does not depend on $n)$.

Throughout the paper we shall use the following conventions concerning vectors and matrices. Given a vector $x=\left(x_{i}\right)$ and a matrix $A=$ $(a(i, j))$ we set $\|x\| \stackrel{\text { def }}{=} \max _{i}\left|x_{i}\right|$, which implies $\|A\|=\sup _{\|x\|=1}\|A x\|=$ $\max _{i} \sum_{j}|a(i, j)|$. We say that $A$ is strictly positive (and write $A>0$ ) if

imsart-aop ver. 2014/10/16 file: InvarMesRWRE.tex date: September 18, 2018 
all its matrix elements satisfy $a(i, j)>0$. $A$ is called non-negative (and we write $A \geq 0$ ), if all $a(i, j)$ are non negative. A similar convention applies to vectors. Note that if $A$ is a non-negative matrix then $\|A\|=\|A \mathbf{1}\|$.

Since $\Omega=\mathcal{J}^{\mathbb{Z}}$, it can be endowed with a metric (in many ways). We shall make use of a metric defined as follows. If $\omega^{\prime}=\left\{\left(P_{n}^{\prime}, Q_{n}^{\prime}, R_{n}^{\prime}\right)\right\}, \omega^{\prime \prime}=$ $\left\{\left(P_{n}^{\prime \prime}, Q_{n}^{\prime \prime}, R_{n}^{\prime \prime}\right)\right\}$ set

$$
\mathbf{d}\left(\omega^{\prime}, \omega^{\prime \prime}\right) \stackrel{\text { def }}{=} \sum_{n \in \mathbb{Z}} \frac{\left\|P_{n}^{\prime}-P_{n}^{\prime \prime}\right\|+\left\|Q_{n}^{\prime}-Q_{n}^{\prime \prime}\right\|+\left\|R_{n}^{\prime}-R_{n}^{\prime \prime}\right\|}{2^{|n|}} .
$$

Below, whenever we say that a function defined on $\Omega$ is continuous, we mean that it is continuous with respect to the topology induce on $\Omega$ by the metric $\mathbf{d}(\cdot, \cdot)$.

The assumptions $\mathbf{C 1}$ and $\mathbf{C 2}$ listed below will be called Condition $\mathbf{C}$.

\section{Condition $\mathbf{C}$}

C1 $\left(P_{n}, Q_{n}, R_{n}\right),-\infty<n<\infty$, is an ergodic sequence (equivalently, $T$ is an ergodic transformation of $\Omega$ ).

C2 There is an $\varepsilon>0$ and a positive integer number $k_{0}<\infty$ such that for any $(P, Q, R) \in \mathcal{J}_{0}$ and all $i, j \in[1, m]$

$$
\left\|R^{k_{0}}\right\| \leq 1-\varepsilon, \quad\left((I-R)^{-1} P\right)(i, j) \geq \varepsilon, \quad\left((I-R)^{-1} Q\right)(i, j) \geq \varepsilon .
$$

REMARK 2.1. The ellipticity condition (2.6) is sufficient for our purposes. It can be weakened but then many statements and proofs become more technical and less transparent which we prefer to avoid. So, C2 is always supposed to be satisfied by all the environments considered in this paper.

Unlike C2, Condition C1 is needed only when random environments are considered and in all such cases it is supposed to be satisfied.

2.2. Environment viewed from the particle and the invariant density equation.

DeFinition 2.2. The environment viewed from the particle is a random process $\left(\bar{\omega}_{t}, Y_{t}\right)$ taking values in $\Omega \times[1, \ldots, m]$ and defined by

$$
\left(\bar{\omega}_{t}, Y_{t}\right)=\left(T^{X_{t}} \omega, Y_{t}\right), t \geq 0
$$

where $X_{t} \in \mathbb{Z}, Y_{t} \in[1, \ldots, m]$ are the coordinates of the process $\xi_{t}=\left(X_{t}, Y_{t}\right)$ defined in Section 2.1 
$\left(\bar{\omega}_{t}, Y_{t}\right)$ is a Markov chain with the phase space $\bar{\Omega} \stackrel{\text { def }}{=} \Omega \times[1, \ldots, m]$ and the transition kernel

$$
(K f)(\omega, i) \stackrel{\text { def }}{=} \sum_{k \in\{-1,0,1\}, 1 \leq j \leq m} \mathcal{Q}_{\omega}((0, i),(k, j)) f\left(T^{k} \omega, j\right),
$$

where $\mathcal{Q}_{\omega}(\cdot, \cdot)$ is defined by $(2.2)$ and $f$ is a bounded measurable function on $\bar{\Omega}$. Note that our notation emphasizes the dependence of the kernel on $\omega$ which is important (as in (2.3)).

We can now explain more precisely the main goal of this work: we want to establish, in terms of the parameters of the environment, the necessary and sufficient conditions for the existence of an invariant measure for the Markov chain (2.7) which is absolutely continuous with respect to the measure $\overline{\mathbb{P}} \stackrel{\text { def }}{=}$ $\mathbb{P} \times\left\{m^{-1}\right\}$ on $\bar{\Omega}$ where $\left\{m^{-1}\right\}$ is the uniform distribution on $[1, \ldots, m]$.

So, suppose that the Markov chain $\left(\bar{\omega}_{t}, Y_{t}\right)$ has an absolutely continuous invariant measure and let $\rho: \bar{\Omega} \mapsto \mathbb{R}$ be the density function of this measure. It is convenient to introduce a row vector $\boldsymbol{\rho}=\boldsymbol{\rho}(\omega)=(\rho(\omega, 1), \ldots, \rho(\omega, m))$, where the component $\rho(\omega, i)$ is the value of the density at $(\omega, i)$.

Lemma 2.3. A non-negative function $\rho: \bar{\Omega} \mapsto \mathbb{R}$ is a density function of the invariant measure of the Markov chain $\left(\bar{\omega}_{t}, Y_{t}\right)$ if and only if the following conditions are satisfied. For all $n \in \mathbb{Z}$

$$
\boldsymbol{\rho}_{n}(\omega)=\boldsymbol{\rho}_{n+1}(\omega) Q_{n+1}(\omega)+\boldsymbol{\rho}_{n}(\omega) R_{n}(\omega)+\boldsymbol{\rho}_{n-1}(\omega) P_{n-1}(\omega),
$$

where

$$
\boldsymbol{\rho}_{n}(\omega) \stackrel{\text { def }}{=} \boldsymbol{\rho}\left(T^{n} \omega\right)
$$

and

$$
\mathbb{E}\left(\sum_{i=1}^{m} \rho(\omega, i)\right)=m
$$

REMARK 2.4. Let us extend the definition of the metric (2.5) to $\bar{\Omega}$ by setting $\overline{\mathbf{d}}\left(\left(\omega^{\prime}, i^{\prime}\right),\left(\omega^{\prime \prime}, i^{\prime \prime}\right)\right) \stackrel{\text { def }}{=} \mathbf{d}\left(\omega^{\prime}, \omega^{\prime \prime}\right)+\left|i^{\prime}-i^{\prime \prime}\right|$. Since $\bar{\Omega}$ is a compact metric space, the Markov chain $\left(\bar{\omega}_{t}, Y_{t}\right)$ has at least one invariant probability measure which, however, may be singular with respect to the measure $\mathbb{P}$. For example, in the transient case absolutely continuous invariant measure exists iff the walk has positive speed (see Theorem 6.12 below). On the other hand we will see in Section 6.3 (cf Remark 6.11) that in the transient case the invariant density equation (2.9) always admits a stationary solution which, in general, may fail to be integrable.

imsart-aop ver. 2014/10/16 file: InvarMesRWRE.tex date: September 18, 2018 
REMARK 2.5. The invariant measure (not density!) of a Markov chain on the strip in a fixed environment (not on the space of environments!) also satisfies equation (2.9) which is a standard textbook statement. In that capacity, this equation was studied in [3] and played a major role in the proof of [3, Lemma 3] (Lemma 3.4 below; see also the proof of Lemma 4.4).

The situation is very different when $\boldsymbol{\rho}$ is interpreted as a density. Namely, we need the stationarity of the environment to derive (2.9) and (2.10). The idea of the proof of Lemma 2.3 goes back to [22] and yet we prove this lemma because, first of all, the proof is short; secondly, it makes our work more self-contained; finally and mainly because it is natural to provide a derivation of an equation which is the main subject of the work.

REMARK 2.6. By (2.10) and (2.11), the sequence $\boldsymbol{\rho}_{n}$ must be stationary and integrable and therefore must satisfy the following standard estimate: for $\mathbb{P}$-a.a. $\omega$ there is a constant $C(\omega)$ such that for all $n$

$$
\left\|\boldsymbol{\rho}_{n}(\omega)\right\| \leq C(\omega)(|n|+1) .
$$

However, as has already been mentioned in section 1.2, the method we use allows us to describe a much wider class of solutions to (2.9), namely all tempered solutions. This will now be done in sections $3,4,5$.

\section{Some preparatory facts and results.}

3.1. Matrices $\zeta_{n}, A_{n}, \alpha_{n}$ and related quantities. We recall the definitions of several objects most of which were first introduced and studied in [3], [4], and which will play a crucial role also in this work.

For a given $\omega \in \Omega$, define a sequence of $m \times m$ stochastic matrices $\zeta_{n}$ as follows. For an integer $a$, let $\psi_{a}$ be a stochastic matrix. For $n>a$ define matrices $\psi_{n}$ recurrently:

$$
\psi_{n}=\left(I-R_{n}-Q_{n} \psi_{n-1}\right)^{-1} P_{n}, \quad n=a+1, a+2, \ldots
$$

It is easy to show ([3]) that matrices $\psi_{n}$ are stochastic. Now for a fixed $n$ set

$$
\zeta_{n}=\lim _{a \rightarrow-\infty} \psi_{n}
$$

By [3, Theorem 1], the limit (3.2) exists and is independent of the choice of the sequence $\psi_{a}$. Moreover, this result implies that the sequence $\zeta_{n}, n \in \mathbb{Z}$, can be defined as the unique sequence of stochastic matrices satisfying the following infinite system of equations:

$$
\zeta_{n}=\left(I-R_{n}-Q_{n} \zeta_{n-1}\right)^{-1} P_{n}, \quad n \in \mathbb{Z} .
$$

imsart-aop ver. 2014/10/16 file: InvarMesRWRE.tex date: September 18, 2018 
The following notation will be useful for the future references:

$$
\gamma_{n} \stackrel{\text { def }}{=}\left(I-R_{n}-Q_{n} \zeta_{n-1}\right)^{-1} .
$$

Next, define probability row-vectors $y_{n}=y_{n}(\omega)=\left(y_{n}(\omega, 1), \ldots, y_{n}(\omega, m)\right)$ by

$$
y_{n} \stackrel{\text { def }}{=} \lim _{a \rightarrow-\infty} \tilde{y}_{a} \zeta_{a} \ldots \zeta_{n-1}
$$

where $\tilde{y}_{a}$ is any sequence of probability row-vectors (that is $\tilde{y}_{a} \geq 0$ and $\left.\sum_{i=1}^{m} \tilde{y}_{a}(i)=1\right)$. By [15, Lemma 1], this limit exists and does not depend on the choice of the sequence $\tilde{y}_{a}$. This fact implies that $y_{n}$ is the unique sequence of probability vectors satisfying the infinite system of equations

$$
y_{n}=y_{n-1} \zeta_{n-1}, \quad n \in \mathbb{Z}
$$

Set

$$
\alpha_{n}=Q_{n+1} \gamma_{n}, \quad A_{n}=\gamma_{n} Q_{n} .
$$

Two more sequences of vectors, $v_{n}$ and $l_{n}$, are defined as follows. By $[4$, Theorem 4], for any sequence of (column) vectors $\tilde{v}_{a} \geq 0, \tilde{v}_{a} \neq 0$, the following limit exists and does not depend on the sequence $\tilde{v}_{a}$ :

$$
v_{n} \stackrel{\text { def }}{=} \lim _{a \rightarrow-\infty} \frac{A_{n} A_{n-1} \ldots A_{a+1} \tilde{v}_{a}}{\left\|A_{n} A_{n-1} \ldots A_{a+1} \tilde{v}_{a}\right\|} .
$$

Similarly, for any sequence of row-vectors $\tilde{l}_{a} \geq 0$ satisfying $\tilde{l}_{a} \alpha_{a-1} \neq 0$, the following limit exists and does not depend on the choice of the sequence $\tilde{l}_{a}$ :

$$
l_{n} \stackrel{\text { def }}{=} \lim _{a \rightarrow \infty} \frac{\tilde{l}_{a} \alpha_{a-1} \alpha_{a-2} \ldots \alpha_{n}}{\left\|\tilde{l}_{a} \alpha_{a-1} \alpha_{a-2} \ldots \alpha_{n}\right\|} .
$$

Set

$$
\lambda_{n}=\left\|A_{n} v_{n-1}\right\| \text { and } \tilde{\lambda}_{n}=\left\|l_{n+1} \alpha_{n}\right\|
$$

then obviously

$$
l_{n+1} \alpha_{n}=\tilde{\lambda}_{n} l_{n}, \quad A_{n} v_{n-1}=\lambda_{n} v_{n}
$$

and for any $n \geq k$ we have

$$
\left\|A_{n} A_{n-1} \ldots A_{k} v_{k-1}\right\|=\lambda_{n} \ldots \lambda_{k}, \quad\left\|l_{n+1} \alpha_{n} \alpha_{n-1} \ldots \alpha_{k}\right\|=\tilde{\lambda}_{n} \ldots \tilde{\lambda}_{k} .
$$

imsart-aop ver. 2014/10/16 file: InvarMesRWRE.tex date: September 18, 2018 
REMARK 3.1. It should be emphasized that the proofs provided in [3], [4] of the existence of the limits (3.2) and (3.8) are in fact working for all (and not just almost all) sequences $\omega$ satisfying (2.6). In particular, (3.8) is a deterministic statement which follows from two facts:

$$
\left\|A_{n}\right\| \leq \text { Const and } A_{n}(i, j) \geq \varepsilon \text { for all } 1 \leq i, j \leq m,
$$

where $\varepsilon$ is the same as in (2.6) and the Const depends only on $m$ and $\varepsilon$. For the proof of these inequalities see [4, Lemmas $1-4])$.

Next, we define

$$
\begin{array}{ll}
\zeta(\omega)=\zeta_{0}(\omega), & A(\omega)=A_{0}(\omega), \quad \alpha(\omega)=\alpha_{0}(\omega), \\
v(\omega)=v_{0}(\omega), & l(\omega)=l_{0}(\omega) \quad \lambda(\omega)=\lambda_{0}(\omega), \quad \tilde{\lambda}(\omega)=\tilde{\lambda}_{0}(\omega)
\end{array}
$$

then

$$
\begin{aligned}
& \zeta_{n}=\zeta\left(T^{n} \omega\right), \quad A_{n}=A\left(T^{n} \omega\right), \quad \alpha_{n}=\alpha\left(T^{n} \omega\right), \\
& v_{n}=v\left(T^{n} \omega\right), \quad l_{n}=l\left(T^{n} \omega\right), \quad \lambda_{n}=\lambda\left(T^{n} \omega\right), \quad \tilde{\lambda}_{n}=\tilde{\lambda}\left(T^{n} \omega\right) .
\end{aligned}
$$

Moreover, the functions $\zeta(\cdot), v(\cdot), l(\cdot)$ are continuous in $\omega$. The continuity of all other functions is implied by the continuity of $\zeta, v$, and $l$. In fact, a stronger result was proved in [11]: the above functions are Hölder continuous with respect to the metric $\mathbf{d}$ defined by (2.5). This regularity plays important role in our analysis.

REMARK 3.2. The case $m=1$ corresponds to the RW on $\mathbb{Z}$ with jumps to the nearest neighbours and the above formulae become very simple. Namely, $\psi_{n}=\zeta_{n}=1, v_{n}=l_{n}=1, A_{n}=\lambda_{n}=\frac{q_{n}}{p_{n}}, \alpha_{n}=\tilde{\lambda}_{n}=\frac{q_{n+1}}{p_{n}}$, etc.

3.2. Matrices $\zeta_{n}^{-}, A_{n}^{-}, \alpha_{n}^{-}$and related quantities. In the above considerations, matrices $P_{n}$ and $Q_{n}$ play asymmetric roles and it turns out to be useful to 'symmetrize' the situation. Namely, let us introduce stochastic matrices $\zeta_{n}^{-}$as the unique sequence of stochastic matrices satisfying the system of equations which is symmetric to (3.3):

$$
\zeta_{n}^{-}=\gamma_{n}^{-} Q_{n},-\infty<n<+\infty
$$

where

$$
\gamma_{n}^{-}=\left(I-R_{n}-P_{n} \zeta_{n+1}^{-}\right)^{-1}
$$

Next we set

$$
A_{n}^{-} \stackrel{\text { def }}{=} \gamma_{n}^{-} P_{n}, \quad \alpha_{n}^{-}=P_{n-1} \gamma_{n}^{-} .
$$

imsart-aop ver. 2014/10/16 file: InvarMesRWRE.tex date: September 18, 2018 
All other related objects are introduced similarly.

Matrices $\zeta_{n}^{-}, \alpha_{n}^{-}, A_{n}^{-}$, etc have properties which are similar to those of matrices $\zeta_{n}, \alpha_{n}, A_{n}$ etc listed above. All these objects will be used below without further explanations.

3.3. Lyapunov exponents and the recurrence and transience criteria revisited. The top Lyapunov exponent of the products of matrices $A_{n}$ and of matrices $A_{n}^{-}$are defined respectively by

$\boldsymbol{\lambda}^{+} \stackrel{\text { def }}{=} \lim _{n \rightarrow \infty} \frac{1}{n} \log \left\|A_{n} A_{n-1} \ldots A_{1}\right\| \quad$ and $\quad \boldsymbol{\lambda}^{-} \stackrel{\text { def }}{=} \lim _{n \rightarrow-\infty} \frac{1}{|n|} \log \left\|A_{n}^{-} A_{n+1}^{-} \ldots A_{-1}^{-}\right\|$.

It follows from (3.12) that

$$
\lambda^{+}=\mathbb{E}\left(\log \left\|A_{k} v_{k-1}\right\|\right)=\mathbb{E}(\log \lambda) .
$$

Next, note that

$$
\alpha_{n} \alpha_{n-1} \ldots \alpha_{1}=Q_{n+1} A_{n} A_{n-1} \ldots A_{2} \gamma_{1}
$$

and therefore

$\boldsymbol{\lambda}^{+}=\lim _{n \rightarrow \infty} \frac{1}{n} \log \left\|\alpha_{n} \ldots \alpha_{1}\right\| \quad$ and similarly $\quad \boldsymbol{\lambda}^{-}=\lim _{n \rightarrow-\infty} \frac{1}{|n|} \log \left\|\alpha_{n}^{-} \ldots \alpha_{-1}^{-}\right\|$.

REMARK 3.3. Formulae (3.20) and (3.22) are due to the following observation. The ellipticity condition (2.6) together with (3.21) imply that there exists a constant $\bar{K}$ such that for each $n \in \mathbb{N}$ the ratio of any two among the expressions $(i)-(i v)$ below is between $1 / \bar{K}$ and $\bar{K}$

$$
\text { (i) }\left\|A_{n} \ldots A_{0}\right\|, \quad(i i)\left\|\alpha_{n} \ldots \alpha_{0}\right\|, \quad(i i i) \prod_{k=0}^{n} \lambda_{k} \quad(i v) \prod_{k=0}^{n} \tilde{\lambda}_{k}
$$

This equivalence will play an important role throughout the paper.

A very important symmetry property of Lyapunov exponents was proved in $[3]$ :

LEMma 3.4. [3, Lemma 3]

$$
\lambda^{+}+\lambda^{-}=0
$$

We need the following deterministic(!) recurrence criterion which we prove in Appendix B.

imsart-aop ver. 2014/10/16 file: InvarMesRWRE.tex date: September 18, 2018 
THEOREM 3.5. Suppose that an environment $\omega=\left\{\left(P_{n}, Q_{n}, R_{n}\right)\right\}_{n \in \mathbb{Z}}$ satisfies (2.6) then:

(a) $\mathcal{P}_{\omega, z}\left(X_{t} \rightarrow+\infty\right.$ as $\left.t \rightarrow+\infty\right)>0$ iff

$$
\sum_{n=1}^{\infty}\left\|A_{n} \ldots A_{1}\right\|<\infty
$$

(b) $\mathcal{P}_{\omega, z}\left(X_{t} \rightarrow-\infty\right.$ as $\left.t \rightarrow+\infty\right)>0$ iff $\sum_{n=0}^{\infty}\left\|A_{-n}^{-} \ldots A_{0}^{-}\right\|<\infty$.

For stationary ergodic environment we recover the following result from [3].

Theorem 3.6 ([3], Theorem 2.). Suppose that Condition $\mathbf{C}$ is satisfied. Then for $\mathbb{P}$-almost all $\omega$ the following holds:

$R W$ is recurrent, that is $\mathcal{P}_{\omega, z}\left(\liminf _{t \rightarrow \infty} X_{t}=-\infty\right.$ and $\lim \sup _{t \rightarrow \infty} X_{t}=$ $\infty)=1$, iff $\boldsymbol{\lambda}^{+}=0$

$R W$ is transient to the right, that is $\mathcal{P}_{\omega, z}\left(X_{t} \rightarrow+\infty\right.$ as $\left.t \rightarrow \infty\right)=1$, iff $\boldsymbol{\lambda}^{+}<0$,

$R W$ is transient to the left, that is $\mathcal{P}_{\omega, z}\left(X_{t} \rightarrow-\infty\right.$ as $\left.t \rightarrow \infty\right)=1$, iff $\lambda^{+}>0$.

Indeed, if $\boldsymbol{\lambda}^{+}<0$ then the terms of the first series in Theorem 3.5 decay exponentially and the terms of the second series grow exponentially. Hence $X_{t} \rightarrow+\infty$ with probability 1 . Likewise, if $\boldsymbol{\lambda}^{+}>0$ then $X_{t} \rightarrow-\infty$ with probability 1. Finally if $\boldsymbol{\lambda}^{+}=0$ then the terms of either series do not tend to 0 due to [19].

4. The deterministic analysis of the invariant measure equation. As has been mentioned above, we start with the deterministic environment. Namely, consider the equation

$$
\pi_{n}=\pi_{n+1} Q_{n+1}+\pi_{n} R_{n}+\pi_{n-1} P_{n-1}, \quad-\infty<n<\infty,
$$

where the sequence $\left(P_{n}, Q_{n}, R_{n}\right)$ is fixed.

We have changed the notation $\boldsymbol{\rho}_{n}$ in (2.9) to $\pi_{n}$ in (4.1) in order to distinguish the properties of the deterministic solutions to (4.1) from those where the dependence on $\omega$ is important. We need the following

DeFinition 4.1. A solution $\pi_{n}$ is tempered if $\lim _{|n| \rightarrow \infty} \frac{\ln \left\|\pi_{n}\right\|}{|n|}=0$.

To an extent, equations (4.1) were analyzed in [3, section 3] and the deterministic result of Lemma 4.4 below, though not stated explicitly in [3],

imsart-aop ver. 2014/10/16 file: InvarMesRWRE.tex date: September 18, 2018 
is hidden inside the proof of Lemma 3 from this work. We shall prove some parts of Lemma 4.4 and hint on how to do the rest. This makes our paper more self-contained and the parts we prove are useful for what follows.

LEMMA 4.2. If $\omega$ satisfies (2.6) and $\pi_{n}$ is a sequence of vectors such that for all $n, \pi_{n}=\pi_{n+1} \alpha_{n}$ (or for all $n, \pi_{n+1}=\pi_{n} \alpha_{n+1}^{-}$) then $\pi_{n}$ solves (4.1).

REMARK 4.3. Condition (2.6) is not used in the proof of this (and the next) lemma in any explicit way. However, it is needed to ensure the existence of the matrices $\alpha_{n}$ and their properties on which the proof depends.

Proof. It follows from (3.3) and (3.7) that

$$
\alpha_{n} P_{n}=Q_{n+1} \gamma_{n} P_{n}=Q_{n+1} \zeta_{n}
$$

and hence

$$
\alpha_{n}=Q_{n+1} \gamma_{n}
$$

Let us now substitute $\pi_{n}=\pi_{n+1} \alpha_{n}$ and $\pi_{n-1}=\pi_{n+1} \alpha_{n} \alpha_{n-1}$ into (4.1). We get

$$
\begin{aligned}
& \pi_{n+1} Q_{n+1}+\pi_{n} R_{n}+\pi_{n-1} P_{n-1} \\
& =\pi_{n+1}\left(Q_{n+1}+\alpha_{n} R_{n}+\alpha_{n} \alpha_{n-1} P_{n-1}\right)=\pi_{n+1} \alpha_{n}=\pi_{n},
\end{aligned}
$$

where we use $Q_{n+1}+\alpha_{n} R_{n}+\alpha_{n} \alpha_{n-1} P_{n-1}=\alpha_{n}$ which is equivalent to (4.3).

Similarly, if $\pi_{n+1}=\pi_{n} \alpha_{n+1}^{-}$then the sequence of vectors $\pi_{n}$ solves (4.1).

LEMMA 4.4. If $\omega$ satisfies (2.6) then equations (4.1) have a unique positive solution which has the following properties:

(i) $\left\|\pi_{0}\right\|=1$

(ii) for all $n$ one has

$$
\pi_{n}=\pi_{n+1} \alpha_{n}, \quad \pi_{n+1}=\pi_{n} \alpha_{n+1}^{-} .
$$

Proof. Step 1. Let us show that non-negative solutions satisfying (4.4) exist and are unique. Obviously, we need to do that only for one of these relations, say $\pi_{n}=\pi_{n+1} \alpha_{n}$. Set

$$
\pi_{n}= \begin{cases}\frac{l_{n}}{\left\|l_{n} \alpha_{n-1} \ldots \alpha_{0}\right\|} & \text { if } n \geq 0 \\ l_{0} \alpha_{-1} \ldots \alpha_{n} & \text { if } n<0\end{cases}
$$

imsart-aop ver. 2014/10/16 file: InvarMesRWRE.tex date: September 18, 2018 
where $l_{n}$ is defined in (3.9). Note that then $\pi_{0}=l_{0}$ and for $n \geq 0$ we have

$$
\pi_{n+1} \alpha_{n}=\frac{l_{n+1} \alpha_{n}}{\left\|l_{n+1} \alpha_{n} \alpha_{n-1} \ldots \alpha_{0}\right\|}=\frac{\tilde{\lambda}_{n} l_{n}}{\tilde{\lambda}_{n} \tilde{\lambda}_{n-1} \ldots \tilde{\lambda}_{0}}=\frac{l_{n}}{\left\|l_{n} \alpha_{n-1} \ldots \alpha_{0}\right\|}=\pi_{n},
$$

where the above equalities follow from (3.11) and (3.12). In the case $n<0$, the relation (4.4) for $\pi_{n}$ is straightforward.

Step 2. The proof of uniqueness of $\pi_{n}$. Note first that the $\pi_{n}$ defined in (4.5) is collinear to $l_{n}$. Indeed, if $n \geq 0$ then this is obvious and if $n<0$ then $l_{0} \alpha_{-1} \ldots \alpha_{n}=\tilde{\lambda}_{-1} \ldots \tilde{\lambda}_{n} l_{n}$ by $(3.11)$. Hence $l_{n}=\pi_{n} /\left\|\pi_{n}\right\|$.

Suppose now that $\tilde{\pi}_{n}$ is another sequence of positive vectors such that $\tilde{\pi}_{n}=\tilde{\pi}_{n+1} \alpha_{n}$ for all $n \in \mathbb{Z}$ and $\left\|\tilde{\pi}_{0}\right\|=1$. Then $\tilde{\pi}_{n}=\tilde{\pi}_{a} \alpha_{a-1} \ldots \alpha_{n}$ for any $a>n$ and hence

$$
\tilde{\pi}_{n} /\left\|\tilde{\pi}_{n}\right\|=\frac{\tilde{\pi}_{a} \alpha_{a-1} \ldots \alpha_{n}}{\left\|\tilde{\pi}_{a} \alpha_{a-1} \ldots \alpha_{n}\right\|}
$$

According to (3.9), the limit of the right hand side of this equality as $a \rightarrow \infty$ is $l_{n}$. Hence $\tilde{\pi}_{n} /\left\|\tilde{\pi}_{n}\right\|=l_{n}$. But then $\tilde{\pi}_{n}=\pi_{n}$.

Similar argument applied to matrices $\alpha_{n}^{-}$proves the existence and uniqueness of a non-negative sequence $\pi_{n}^{-}$such that $\left\|\pi_{0}^{-}\right\|=1$ and $\pi_{n+1}^{-}=\pi_{n}^{-} \alpha_{n+1}^{-}$.

Step 3. To finish the proof of Lemma 4.4, it remains to check that $\pi_{n}=\pi_{n}^{-}$.

The detailed proof of this fact can be found in [3]. Here, we briefly explain a slightly modified version of the proof from this work.

Restrict the original Markov chain $\xi_{t}$ to the box $a \leq n \leq b$ with reflecting boundary conditions. To do this, replace the triples $\left(P_{a}, Q_{a}, R_{a}\right)$ and $\left(P_{b}, Q_{b}, R_{b}\right)$ by $\left(\tilde{P}_{a}, \tilde{Q}_{a}, \tilde{R}_{a}\right)=\left(\zeta_{a}, 0,0\right)$ and $\left(\tilde{P}_{b}, \tilde{Q}_{b}, \tilde{R}_{b}\right)=\left(0, \zeta_{b}^{-}, 0\right)$. Present the invariant measure of this chain as a sequence of vectors $\left(\hat{\pi}_{n}\right)_{a \leq n \leq b}$, where $\hat{\pi}_{n}=\left(\hat{\pi}_{n}(i)\right)_{1 \leq i \leq m}$ and $\hat{\pi}_{n}(i)=P_{\omega}\left(\xi_{0}=(n, i)\right)$.

Vectors $\hat{\pi}_{n}$ satisfy (4.1) with boundary conditions $\hat{\pi}_{a}=\hat{\pi}_{a+1} Q_{a+1}, \hat{\pi}_{b}=$ $\hat{\pi}_{b-1} P_{b-1}$. Next, show that there is a solution $\hat{\pi}_{n}$ for the invariant measure of the Markov chain such that $\hat{\pi}_{n}=\hat{\pi}_{n+1} \alpha_{n}$ for $a \leq n \leq b-1$. Since the Markov chain on a finite box has a unique invariant measure, this solution also satisfies $\hat{\pi}_{n+1}=\hat{\pi}_{n} \alpha_{n+1}^{-}$when $a+1 \leq n \leq b$. Finally, consider the sequence $\tilde{\pi}_{n}=\frac{\hat{\pi}_{n}}{\left\|\hat{\pi}_{0}\right\|}$ and pass to the limit $a \rightarrow-\infty, b \rightarrow \infty$. It is easy to see that such a limit exists and the obtained sequence coincides with $\pi_{n}$ and $\pi_{n}^{-}$.

Our next lemma is purely algebraic.

Lemma 4.5. Suppose that a sequence $\pi_{n}, n \in \mathbb{Z}$, solves (4.1). Define vectors $h_{n}$ and $h_{n}^{-}$by

$$
h_{n} \stackrel{\text { def }}{=} \pi_{n}-\pi_{n+1} \alpha_{n}, \quad h_{n}^{-} \stackrel{\text { def }}{=} \pi_{n}-\pi_{n-1} \alpha_{n}^{-} .
$$

imsart-aop ver. 2014/10/16 file: InvarMesRWRE.tex date: September 18, 2018 
Then the following equations hold:

$$
h_{n}=h_{n-1} P_{n-1} \gamma_{n}, \quad h_{n}^{-}=h_{n+1}^{-} Q_{n+1} \gamma_{n}^{-},
$$

Proof. Due to (4.3) we can present $Q_{n+1}=\alpha_{n}-\alpha_{n} R_{n}-\alpha_{n} \alpha_{n-1} P_{n-1}$. Then (4.1) implies

$$
\pi_{n}=\pi_{n+1}\left(\alpha_{n}-\alpha_{n} R_{n}-\alpha_{n} \alpha_{n-1} P_{n-1}\right)+\pi_{n} R_{n}+\pi_{n-1} P_{n-1}
$$

which can be rearranged as

$$
\pi_{n}-\pi_{n+1} \alpha_{n}=\left(\pi_{n}-\pi_{n+1} \alpha_{n}\right) R_{n}+\left(\pi_{n}-\pi_{n+1} \alpha_{n}\right) \alpha_{n-1} P_{n-1}+\left(\pi_{n-1}-\pi_{n} \alpha_{n-1}\right) P_{n-1} .
$$

We thus have

$$
h_{n}=h_{n} R_{n}+h_{n} \alpha_{n-1} P_{n-1}+h_{n-1} P_{n-1} .
$$

By $(4.2), \alpha_{n-1} P_{n-1}=Q_{n} \zeta_{n-1}$ and so $h_{n}\left(I-R_{n}-Q_{n} \zeta_{n-1}\right)=h_{n-1} P_{n-1}$. Hence $h_{n}=h_{n-1} P_{n-1}\left(I-R_{n}-Q_{n} \zeta_{n-1}\right)^{-1}=h_{n-1} P_{n-1} \gamma_{n}$.

The second relation is proved similarly.

The following lemma describes a fundamental property of equation (4.1): this equation has a first integral.

Lemma 4.6. The quantities

$$
\mathfrak{c}=h_{n} P_{n} \mathbf{1} \text { and } \mathfrak{c}^{-}=h_{n}^{-} Q_{n} \mathbf{1}
$$

do not depend on $n$ (are preserved by equations (4.1)). Moreover

$$
\mathfrak{c}=\pi_{n} P_{n} \mathbf{1}-\pi_{n+1} Q_{n+1} \mathbf{1} \text { and } \mathfrak{c}=-\mathfrak{c}^{-} .
$$

Proof. We have

$$
h_{n} P_{n}=h_{n-1} P_{n-1} \gamma_{n} P_{n}=h_{n-1} P_{n-1} \zeta_{n}
$$

where the first equality follows by (4.7) and the second equality follows by (3.3). Since $\zeta_{n}$ is a stochastic matrix we get

$$
h_{n} P_{n} \mathbf{1}=h_{n-1} P_{n-1} \zeta_{n} \mathbf{1}=h_{n-1} P_{n-1} \mathbf{1}
$$

which proves the first statement in (4.8). Similarly, $\gamma_{n}^{-} Q_{n}=\zeta_{n}^{-}$is stochastic and hence

$$
h_{n}^{-} Q_{n} \mathbf{1}=h_{n+1}^{-} Q_{n+1} \gamma_{n}^{-} Q_{n} \mathbf{1}=h_{n+1}^{-} Q_{n+1} \mathbf{1}
$$

imsart-aop ver. 2014/10/16 file: InvarMesRWRE.tex date: September 18, 2018 
Let us now turn to (4.9). It follows from (4.6) and (4.2) that

$$
\begin{aligned}
\mathfrak{c} & =h_{n} P_{n} \mathbf{1}=\pi_{n} P_{n} \mathbf{1}-\pi_{n+1} \alpha_{n} P_{n} \mathbf{1} \\
& =\pi_{n} P_{n} \mathbf{1}-\pi_{n+1} Q_{n+1} \zeta_{n} \mathbf{1}=\pi_{n} P_{n} \mathbf{1}-\pi_{n+1} Q_{n+1} \mathbf{1}
\end{aligned}
$$

This proves the first relation in (4.9).

Next, by (4.6), $h_{n+1}^{-}=\pi_{n+1}-\pi_{n} \alpha_{n+1}^{-}$and hence

$$
\mathfrak{c}^{-}=h_{n+1}^{-} Q_{n+1} \mathbf{1}=\pi_{n+1} Q_{n+1} \mathbf{1}-\pi_{n} \alpha_{n+1}^{-} Q_{n+1} \mathbf{1} .
$$

It follows from (3.16) and (3.18) that $\alpha_{n+1}^{-} Q_{n+1}=P_{n} \zeta_{n+1}^{-}$. Therefore $\mathfrak{c}^{-}=\pi_{n+1} Q_{n+1} \mathbf{1}-\pi_{n} P_{n} \zeta_{n+1}^{-} \mathbf{1}=\pi_{n+1} Q_{n+1} \mathbf{1}-\pi_{n} P_{n} \mathbf{1}=-\mathfrak{c}$.

Definition 4.7. A solution $\pi_{n}$ is monotone increasing if $h_{n} P_{n} \geq 0$ and it is monotone decreasing if $h_{n} P_{n} \leq 0$. If these inequalities are strict then we say that $\pi_{n}$ is strictly monotone increasing or strictly monotone decreasing.

Lemma 4.8. Suppose that condition (2.6) is satisfied and that vectors $\pi_{n}, n \in \mathbb{Z}$, solve (4.1) and $\pi_{n}$ is either tempered or monotone. Then

$$
h_{n}=\mathfrak{c} y_{n} \gamma_{n}, \quad h_{n}^{-}=\mathfrak{c}^{-} y_{n}^{-} \gamma_{n}^{-}
$$

Proof. (a) Suppose that $\pi_{n}$ is tempered. Iterating (4.7) we obtain for any $k \geq 1$ that

$$
h_{n}=h_{n-k} P_{n-k} \zeta_{n-k+1} \cdots \zeta_{n-1} \gamma_{n} .
$$

It follows from (3.5) that $\zeta_{n-k+1} \cdots \zeta_{n-1}=\left(y_{n}(1) \mathbf{1}, \ldots y_{n}(m) \mathbf{1}\right)+r_{k}$, where $r_{k}$ is an $m \times m$ matrix with $\left\|r_{k}\right\| \leq C \theta^{k}$ with $C$ and $\theta<1$ depending only on the $\varepsilon$ from condition (2.6). Hence

$$
\begin{aligned}
h_{n} & =h_{n-k} P_{n-k}\left(\left(y_{n}(1) \mathbf{1}, \ldots y_{n}(m) \mathbf{1}\right)+r_{k}\right) \gamma_{n} \\
& =\left(y_{n}(1) h_{n-k} P_{n-k} \mathbf{1}, \ldots y_{n}(m) h_{n-k} P_{n-k} \mathbf{1}\right) \gamma_{n}+h_{n-k} P_{n-k} r_{k} \gamma_{n} \\
& =\mathfrak{c} y_{n} \gamma_{n}+h_{n-k} P_{n-k} r_{k} \gamma_{n}=\mathfrak{c} y_{n} \gamma_{n}+\mathcal{O}\left(e^{(|n|+k) \epsilon} \theta^{k}\right) .
\end{aligned}
$$

Here the term $\mathfrak{c} y_{n} \gamma_{n}$ is due to the equality $h_{n-k} P_{n-k} \mathbf{1}=\mathfrak{c}$ and the estimate $\mathcal{O}(\cdot)$ follows from (4.6) and the fact that $\pi_{n}$ is tempered which imply that

$$
\left\|h_{n-k}\right\|=\left\|\pi_{n-k}-\pi_{n-k+1} \alpha_{n-k}\right\| \leq\left\|\pi_{n-k}\right\|+\left\|\pi_{n-k+1}\right\|\left\|\alpha_{n-k}\right\| \leq C(\epsilon) e^{(|n|+k) \epsilon} .
$$

We now choose $\epsilon>0$ such that $\theta e^{\epsilon}<1$ and pass in (4.14) to the $k \rightarrow \infty$ limit. This gives $h_{n}=\mathfrak{c} y_{n} \gamma_{n}$. 
(b) Suppose that $\pi_{n}$ is, say, monotone increasing. By (4.10), $h_{n} P_{n}=$ $h_{n-1} P_{n-1} \zeta_{n}$. Since $h_{n} P_{n} \mathbf{1}=\mathfrak{c}$, this together with the positivity of $h_{n} P_{n}$ implies that $h_{n} P_{n}=\mathfrak{c} \tilde{z}_{n}$ where $\tilde{z}_{n}$ is a probability vector and $\tilde{z}_{n}=\tilde{z}_{n-1} \zeta_{n}$. Since there is only one sequence of probability vectors satisfying (3.6) we conclude that $\tilde{z}_{n}=y_{n+1}$. Hence, by (4.7), we have that $h_{n}=\mathfrak{c} y_{n} \gamma_{n}$.

REMARK 4.9. In (4.14), neither $h_{n}$ nor $y_{n} \gamma_{n}$ depend on $k$. This proves that in fact $h_{n-k} P_{n-k} r_{k} \gamma_{n}=0$ for all $k \geq 1$ (note that $r_{k}$ depends also on $n$ but our notation ignores this fact as it is not essential for our purposes).

Lemma 4.8 implies the following

Corollary 4.10. If a sequence $\pi_{n}, n \in \mathbb{Z}$ is a tempered solution to (4.1) and $h_{n}=\pi_{n}-\pi_{n+1} \alpha_{n}$ then either all $h_{n}>0$, or all $h_{n}<0$, or all $h_{n}=0$.

REMARK 4.11. The relation $\mathfrak{c}=\pi_{n} P_{n} \mathbf{1}-\pi_{n+1} Q_{n+1} \mathbf{1}$ (and similarly for $\mathfrak{c}^{-}$) shows that $\mathfrak{c}$ and $\mathfrak{c}^{-}$are linear functionals on the space of solutions to (4.1). Since these functionals play symmetric roles, we shall suppose from now on, without loss of generality, that $\mathfrak{c} \geq 0$.

We are now in a position to describe all tempered solutions to (4.1).

Theorem 4.12. Suppose that $\pi_{n}, n \in \mathbb{Z}$, is a tempered sequence of positive vectors solving (4.1). Set $\mathfrak{c}=\pi_{n} P_{n} \mathbf{1}-\pi_{n+1} Q_{n+1} \mathbf{1} \geq 0$. Then:

(i) either $\mathfrak{c}=0$ and the solution $\pi_{n}$ satisfies

$$
\pi_{n}=\pi_{n+1} \alpha_{n}
$$

(ii) or $\mathfrak{c}>0$ and (4.1) has a solution of the form

$$
\bar{\pi}_{n}=\mathfrak{c}\left(\bar{h}_{n}+\bar{h}_{n+1} \alpha_{n}+\ldots+\bar{h}_{n+k} \alpha_{n+k-1} \ldots \alpha_{n}+\ldots\right),
$$

where $\bar{h}_{n}=y_{n} \gamma_{n}$ with $y_{n}$ defined by (3.5).

(iii) The following conditions are equivalent

(A) (4.1) admits a solution of the form (4.15);

(B) The following series converges

$$
\sum_{k=0}^{\infty}\left\|\alpha_{k} \ldots \alpha_{0}\right\|<\infty
$$

(C) $\mathcal{P}_{\omega, z}\left(X_{t} \rightarrow+\infty\right.$ as $\left.t \rightarrow \infty\right)>0$. 
Proof of Theorem 4.12. (i) If $\mathfrak{c}=0$ then all $h_{n}=0$ by Lemma 4.8 and the first statement follows.

(ii) Suppose now that $\mathfrak{c}>0$. Due to Lemma 4.8, we can rewrite (4.6) as

$$
\pi_{n}=\pi_{n+1} \alpha_{n}+h_{n}=\pi_{n+1} \alpha_{n}+\mathfrak{c} y_{n} \gamma_{n} .
$$

Iterating (4.17) gives

$$
\pi_{n}=h_{n}+h_{n+1} \alpha_{n}+\ldots+h_{n+k} \alpha_{n+k-1} \ldots \alpha_{n}+\pi_{n+k+1} \alpha_{n+k} \alpha_{n+k-1} \ldots \alpha_{n}
$$

and hence, because $\pi_{n+k+1} \alpha_{n+k} \alpha_{n+k-1} \ldots \alpha_{n} \geq 0$, we have

$$
\pi_{n} \geq h_{n}+h_{n+1} \alpha_{n}+\ldots+h_{n+k} \alpha_{n+k-1} \ldots \alpha_{n}
$$

Thus the existence of a positive solution $\pi_{n}$ implies that series (4.15) converges. But then $\bar{\pi}_{n}-\alpha_{n} \bar{\pi}_{n+1}=h_{n}$ (direct computation) and hence the sequence $\bar{\pi}_{n}, n \in \mathbb{Z}$, solves (4.1).

(iii) The equivalence of (B) and (C) follows from Theorem 3.5 and Remark 3.3. Therefore, it is sufficient to prove that $(\mathrm{A})$ is equivalent to $(\mathrm{B})$.

Suppose first that series (4.16) converges. Then for $k \geq 0$

$$
\left\|\bar{h}_{n+k} \alpha_{n+k-1} \ldots \alpha_{n}\right\| \leq\left\|\bar{h}_{n+k}\right\|\left\|\alpha_{n+k-1} \ldots \alpha_{n}\right\| \leq \mathrm{C}_{n}\left\|\alpha_{n+k-1} \ldots \alpha_{n}\right\|
$$

where the constant $\mathrm{C}_{n}$ depends only on the $\varepsilon$. The convergence of (4.15) follows.

Suppose now that $\mathfrak{c}>0$ and (4.15) converges. Since $\bar{h}_{n+k}=y_{n+k} \gamma_{n+k} \geq$ $y_{n+k} \geq \varepsilon \mathbf{1}^{*}$, where $\mathbf{1}^{*}$ is a row vector whose all components are 1 , we have

$$
\left\|\bar{h}_{n+k} \alpha_{n+k-1} \ldots \alpha_{n}\right\| \geq \varepsilon\left\|\mathbf{1}^{*} \alpha_{n+k-1} \ldots \alpha_{n}\right\|=\varepsilon\left\|\alpha_{n+k-1} \ldots \alpha_{n}\right\|_{*} .
$$

We use the notation $\|B\|_{*} \stackrel{\text { def }}{=}\left\|B^{*}\right\|$, where $B$ is a matrix. Since all norms in a finite-dimensional space are equivalent, the convergence of (4.16) follows.

REMARK 4.13. (a) Inequality (4.19) implies that $\pi_{n} \geq \bar{\pi}_{n}$. In this sense, $\bar{\pi}_{n}$ from (4.15) is the smallest possible positive solution corresponding to the given $\mathfrak{c}>0$.

(b) Denote $\delta_{n}=\pi_{n}-\bar{\pi}_{n}$. Since $\delta_{n}=\delta_{n+1} \alpha_{n}$ we have

$$
\delta_{n}=\delta_{n+2} \alpha_{n+1} \alpha_{n}=\delta_{n+2} Q_{n+2} \gamma_{n+1} Q_{n+1} \gamma_{n}=\delta_{n+2} Q_{n+2} A_{n+1} \gamma_{n} .
$$

Since $A_{n+1}>0$ and $\gamma_{n} \geq I$ we conclude that if $\delta_{n} \geq 0$ and $\delta_{n} \neq 0$ then $\delta_{n}>0($ for all $n \in \mathbb{Z})$. 
Next, (4.15) and (4.18) imply that for $k \geq 0$ (and any $n$ )

$$
\pi_{n}-\bar{\pi}_{n}=\pi_{n+k+1} \alpha_{n+k} \alpha_{n+k-1} \ldots \alpha_{n}-\mathfrak{c} \sum_{j=k}^{\infty} \bar{h}_{n+j+1} \alpha_{n+j} \ldots \alpha_{n}
$$

Sending $k \rightarrow \infty$ and taking into account the fact that the series in (4.15) converges we obtain

$$
\pi_{n}-\bar{\pi}_{n}=\lim _{k \rightarrow \infty} \pi_{n+k+1} \alpha_{n+k} \alpha_{n+k-1} \ldots \alpha_{n} .
$$

Note that $\left\|\alpha_{n+k} \alpha_{n+k-1} \ldots \alpha_{n}\right\| \rightarrow 0$ as $k \rightarrow \infty$. If now $\pi_{n}-\bar{\pi}_{n}>0$ then we must have that $\left\|\pi_{n+k+1}\right\| \rightarrow \infty$ as $k \rightarrow \infty$ and moreover, $\left\|\pi_{n+k+1}\right\| \sim$ $\left\|\alpha_{n+k} \alpha_{n+k-1} \ldots \alpha_{n}\right\|^{-1}$. So, if we know that $\left\|\alpha_{n+k} \alpha_{n+k-1} \ldots \alpha_{n}\right\|$ decays exponentially in $k$ then the only tempered solution to (4.1) is given by (4.15) (if it exists).

5. Probabilistic analysis of the invariant density equation. We now return to the study of the invariant density equation (2.9) in random environment. In particular, condition $\mathbf{C}$ is assumed throughout this section.

5.1. Positive tempered solutions to (2.9).

Lemma 5.1. If $\boldsymbol{\lambda}^{+}<0$ then for $\mathbb{P}$ a.e. $\omega$, the series (3.24) converges and the solution given by (4.15) is tempered.

Proof. The convergence of (3.24) follows from the fact that the $n$-th term of this sum is exponentially small. To show that the solution given by (4.15) is tempered we consider two cases.

(I) On the left from 0 we use the identity (4.18)

$$
\boldsymbol{\rho}_{-n}=\sum_{k=0}^{n-1} h_{-(n-k)} \alpha_{-(n-k+1)} \ldots \alpha_{-n}+\boldsymbol{\rho}_{0} \alpha_{-1} \ldots \alpha_{-n},
$$

where $n>0$ and we use the convention $\alpha_{-n-1} \alpha_{-n}=I$. By Remark 3.3, we can write

$$
\left\|\boldsymbol{\rho}_{-n}\right\| \leq C \sum_{k=0}^{n} \exp \left(\sum_{j=k}^{n} \ln \lambda_{-j}\right) .
$$

By the ergodic theorem, the expression in the parenthesis equals to

$$
\sum_{j=0}^{n} \ln \lambda_{-j}-\sum_{j=0}^{k} \ln \lambda_{-j}=\lambda^{+}(n-k)+r_{k}+r_{n}
$$

imsart-aop ver. 2014/10/16 file: InvarMesRWRE.tex date: September 18, 2018 
where, for any given $\bar{\varepsilon}>0$, both $\left|r_{n}\right|$ and $\left|r_{k}\right|$ are less than $\bar{\varepsilon} n$ provided that $n$ is large enough. It follows that $\left\|\boldsymbol{\rho}_{-n}\right\| \leq C(n+1) e^{2 \varepsilon n}$. Since $\bar{\varepsilon}$ can be chosen arbitrarily small $\lim _{n \rightarrow \infty} n^{-1} \ln \left\|\boldsymbol{\rho}_{-n}\right\|=0$ as needed.

(II) On the right from 0 we use (4.15) to see that

$$
\left\|\boldsymbol{\rho}_{n}\right\| \leq C \sum_{k=0}^{\infty} \exp \left(\sum_{j=n}^{n+k} \ln \lambda_{j}\right) .
$$

The expression in the parenthesis equals to $k \boldsymbol{\lambda}^{+}+r_{n}+r_{n+k}$ where, for any given $\bar{\varepsilon}$, both $\left|r_{n}\right| \leq \bar{\varepsilon} n$ and $\left|r_{n+k}\right| \leq \bar{\varepsilon}(n+k)$ provided that $n$ is large enough. Considering separately the cases $k<n$ and $k>n$ we get $\left\|\boldsymbol{\rho}_{n}\right\| \leq C e^{3 \bar{\varepsilon} n} n$. Since $\bar{\varepsilon}$ can be chosen to be arbitrarily small $\lim _{n \rightarrow \infty} n^{-1} \ln \left\|\boldsymbol{\rho}_{n}\right\|=0$ as needed.

Our next lemma provides classification of all solutions to (2.9) for general ergodic environments by establishing the relation between the sign of the constant $\mathfrak{c}$ and the sign of the Lyapunov exponent $\boldsymbol{\lambda}^{+}$. Relations (3.23) and (4.9) allow us to consider only the case $\mathfrak{c} \geq 0$.

Lemma 5.2. (The Classification Lemma)

(a) For $\mathbb{P}$ a.e. $\omega$ there exists a unique, up to multiplication by a positive constant, tempered positive solution to (2.9).

(b) $\operatorname{sign}(\mathfrak{c})=-\operatorname{sign}\left(\boldsymbol{\lambda}^{+}\right)$.

(c) If $\mathfrak{c} \neq 0$ then there exists a sequence of functions $\boldsymbol{\rho}_{n}(\omega)$ satisfying both (2.9) and (2.10). In particular, if $\mathfrak{c}>0$ then this solution is given by

$$
\boldsymbol{\rho}_{n}(\omega)=h_{n}+h_{n+1} \alpha_{n}+\ldots+h_{n+k} \alpha_{n+k-1} \ldots \alpha_{n}+\ldots
$$

Proof. By Theorem 4.12, if there is a positive tempered solution to (2.9) with $\mathfrak{c}>0$, then $\xi$ escapes to $+\infty$ with positive probability. By Theorem 3.6 this is only possible if $\boldsymbol{\lambda}^{+}<0$. Conversely, if $\boldsymbol{\lambda}^{+}<0$ then by Lemma 5.1, (2.9) admits a positive tempered solution. Thus a positive tempered solution to $(2.9)$ with $\mathfrak{c}>0$ exists iff $\boldsymbol{\lambda}^{+}<0$. The uniqueness follows from part (ii) of Theorem 4.12.

Likewise, a positive tempered solution to (2.9) with $\mathfrak{c}<0$ exists iff $\boldsymbol{\lambda}^{+}>0$.

Also by Theorem 4.12 , the solution with $\mathfrak{c}=0$ must satisfy

$\boldsymbol{\rho}_{n}(\omega)=\boldsymbol{\rho}_{n+1}(\omega) \alpha_{n}(\omega), \quad \boldsymbol{\rho}_{n+1}(\omega)=\boldsymbol{\rho}_{n}(\omega) \alpha_{n+1}^{-}(\omega)$ for all $n$ and $\mathbb{P}$-a.a. $\omega$.

This solution is tempered iff the Lyapunov exponents of both $\alpha$ and $\alpha^{-}$are non-positive, that is, iff $\boldsymbol{\lambda}^{+}=0$.

This proves parts (a) and (b). To prove part (c) (for $\mathfrak{c}>0$ ) we observe that the solution given by (5.1) is stationary.

imsart-aop ver. 2014/10/16 file: InvarMesRWRE.tex date: September 18, 2018 
5.2. The existence of the invariant density. In Section 4 there was no necessity to emphasize the dependence of the constant $\mathfrak{c}$ on the sequence $\omega$. But for the study of the invariant density the control of the dependence on $\omega$ of the sequence $\boldsymbol{\rho}_{n}(\omega)$ solving (2.9) is crucial. In principle, also $\mathfrak{c}$, as a function of the whole sequence, could depend on $\omega$ in a non-trivial way. However, the following simple observation shows that for stationary solutions of (2.9) this is not so.

Lemma 5.3. Suppose that an $m$-dimensional vector $\boldsymbol{\rho}(\omega): \Omega \rightarrow \mathbb{R}^{m}$ is measurable and for $\mathbb{P}$-almost all $\omega$ the sequence $\boldsymbol{\rho}_{n}(\omega)=\boldsymbol{\rho}\left(T^{n} \omega\right), n \in \mathbb{Z}$, solves (2.9). Then there is a constant $\mathfrak{c}$ such that $h_{n}(\omega) P_{n}(\omega) \mathbf{1}=\mathfrak{c}$ for $\mathbb{P}_{-}$ almost all $\omega$.

Proof. We know from Lemma 4.6 that $h_{n}(\omega) P_{n}(\omega) \mathbf{1}$ does not depend on $n$ for all those $\omega$ for which the sequence $\boldsymbol{\rho}_{n}(\omega)=\boldsymbol{\rho}\left(T^{n} \omega\right), n \in \mathbb{Z}$ solves (2.9). On the other hand,

$h_{n}(\omega)=\boldsymbol{\rho}_{n}(\omega)-\boldsymbol{\rho}_{n+1}(\omega) \alpha_{n}(\omega)=\boldsymbol{\rho}_{0}\left(T^{n} \omega\right)-\boldsymbol{\rho}_{1}\left(T^{n} \omega\right) \alpha_{0}\left(T^{n} \omega\right)=h_{0}\left(T^{n} \omega\right)$.

We thus have $h_{0}(\omega) P_{0}(\omega) \mathbf{1}=h_{1}(\omega) P_{1}(\omega) \mathbf{1}=h_{0}(T \omega) P_{0}(T \omega) \mathbf{1}$. Since $T$ is ergodic, there exists a constant $\mathfrak{c}$ such that $h_{0}(\omega) P_{0}(\omega) \mathbf{1}=\mathfrak{c}$ for $\mathbb{P}$-almost all $\omega$.

We are now ready to describe the necessary and sufficient conditions for the existence of the invariant density. We consider the transient and the recurrent cases separately.

Theorem 5.4. Suppose that condition $\mathbf{C}$ is satisfied and that $\boldsymbol{\lambda}^{+}<0$ (so that $\xi$ is transient to the right). Then

(i) The Markov chain $\left(\bar{\omega}_{t}, Y_{t}\right)$ has the invariant density if and only if

$$
\sum_{n=0}^{\infty} \mathbb{E}\left(\left\|\alpha_{n} \alpha_{n-1} \ldots \alpha_{0}\right\|\right)<\infty .
$$

(ii) The invariant density is unique and is given by

$$
\boldsymbol{\rho}(\omega)=\mathfrak{c}\left(\bar{h}_{0}(\omega)+\bar{h}_{1}(\omega) \alpha_{0}(\omega)+\ldots+\bar{h}_{k}(\omega) \alpha_{k-1}(\omega) \ldots \alpha_{0}(\omega)+\ldots\right)
$$

where $\bar{h}_{k}(\omega)=y_{k}(\omega) \gamma_{k}(\omega)=y_{0}\left(T^{k} \omega\right) \gamma_{0}\left(T^{k} \omega\right), \alpha_{k}(\omega)=\alpha_{0}\left(T^{k} \omega\right)$ and

$$
\mathfrak{c}^{-1}=\mathbb{E}\left(\bar{h}_{0} \mathbf{1}+\bar{h}_{1} \alpha_{0} \mathbf{1}+\ldots+\bar{h}_{k} \alpha_{k-1} \ldots \alpha_{0} \mathbf{1}+\ldots\right) .
$$


Proof. If $\boldsymbol{\lambda}^{+}<0$ then, by the Classification Lemma 5.2, (2.9) has a unique, up to a multiplication by a positive constant, solution which is stationary (satisfies (2.10)). This solution is given by (5.1). The invariant density exists iff this solution is also integrable, $\mathbb{E}(\rho)<\infty$. By (5.1), (4.20), $(4.21)$

$$
\varepsilon \sum_{n=0}^{\infty}\left\|\alpha_{n} \alpha_{n-1} \ldots \alpha_{0}\right\|(\omega) \leq \rho(\omega)<C \sum_{n=0}^{\infty}\left\|\alpha_{n} \alpha_{n-1} \ldots \alpha_{0}\right\|(\omega) .
$$

Thus integrability of $\rho$ is equivalent to (5.3). This proves part (i). Part (ii) is a direct consequence of Lemma 5.2.

Finally, the recurrent case is characterized as follows. We set $\tilde{\lambda}(\omega) \stackrel{\text { def }}{=}$ $\tilde{\lambda}_{0}(\omega)($ see $(3.10))$.

Theorem 5.5. Suppose that $\boldsymbol{\lambda}^{+}=0$ (so that $\xi$ is recurrent). Then the Markov chain $\left(\bar{\omega}_{t}, Y_{t}\right)$ has an invariant density if and only if there is a nonnegative function $\tilde{\beta}: \Omega \mapsto \mathbb{R}$ such that

$$
\tilde{\lambda}(\omega)=\frac{\tilde{\beta}(\omega)}{\tilde{\beta}(T \omega)} \text { for } \mathbb{P} \text {-a.a. } \omega \text { and } \mathbb{E}(\tilde{\beta})<\infty .
$$

Proof. Suppose that (5.6) holds. Then it follows from (3.11) and condition (5.6) that

$$
l_{n+1} \alpha_{n}=\tilde{\lambda}_{n} l_{n}=\frac{\tilde{\beta}\left(T^{n} \omega\right)}{\tilde{\beta}\left(T^{n+1} \omega\right)} l_{n} \text { and so } \tilde{\beta}\left(T^{n+1} \omega\right) l_{n+1} \alpha_{n}=\tilde{\beta}\left(T^{n} \omega\right) l_{n} .
$$

Remember that $l_{n}=l\left(T^{n} \omega\right)($ see $(3.9))$. Set

$$
\boldsymbol{\rho}(\omega)=Z^{-1} \tilde{\beta}(T \omega) l(\omega), \text { where } Z=\mathbb{E}\left[\tilde{\beta}(T \omega) \sum_{i=1}^{m} l(\omega, i)\right] .
$$

Then the second equation in (5.7) can be written, for all $n$, as $\boldsymbol{\rho}_{n-1}=$ $\boldsymbol{\rho}_{n} \alpha_{n-1}$, where $\boldsymbol{\rho}_{n}=\boldsymbol{\rho}\left(T^{n} \omega\right)$. Hence, the sequence $\boldsymbol{\rho}_{n}, n \in \mathbb{Z}$, solves $(2.9)$ and hence $\boldsymbol{\rho}$ defined by (5.8) is the density of the invariant measure of our Markov chain.

Suppose now that $\boldsymbol{\rho}(\omega)$ is a density and the random walk is recurrent. Set $\boldsymbol{\rho}_{n}=\boldsymbol{\rho}\left(T^{n} \omega\right)$. Then the sequence $\left\{\boldsymbol{\rho}_{n}, n \in \mathbb{Z}\right\}$ is a positive tempered solution to (2.9). Since $\boldsymbol{\lambda}=0$ we have, by Lemma 5.2(iii) that $\boldsymbol{\rho}_{n}=\boldsymbol{\rho}_{n+1} \alpha_{n}$. Rewrite this as

$$
\frac{\boldsymbol{\rho}_{n+1}}{\left\|\boldsymbol{\rho}_{n+1}\right\|} \alpha_{n}=\frac{\left\|\boldsymbol{\rho}_{n}\right\|}{\left\|\boldsymbol{\rho}_{n+1}\right\|} \frac{\boldsymbol{\rho}_{n}}{\left\|\boldsymbol{\rho}_{n}\right\|} \text {. }
$$

imsart-aop ver. 2014/10/16 file: InvarMesRWRE.tex date: September 18, 2018 
The last relation is just the same as (3.11) and since there is only one sequence $l_{n}$ satisfying (3.11) we have that

$$
l_{n}=\frac{\boldsymbol{\rho}_{n}}{\left\|\boldsymbol{\rho}_{n}\right\|}, \quad \tilde{\lambda}_{n}=\frac{\left\|\boldsymbol{\rho}_{n}\right\|}{\left\|\boldsymbol{\rho}_{n+1}\right\|} .
$$

We can now set

$$
\tilde{\beta}(\omega)=\left\|\rho_{0}\left(T^{-1} \omega\right)\right\|=\max _{i}\left\{\rho\left(T^{-1} \omega, i\right)\right\} .
$$

(We could have set $\tilde{\beta}(\omega)=\left\|\boldsymbol{\rho}_{0}(\omega)\right\|=\max _{i}\{\rho(\omega, i)\}$ but this would be inconsistent with (5.8).)

\section{Applications.}

6.1. Positive and null recurrence. Recall that an irreducible Markov chain (MC) is positive recurrent if it satisfies the following equivalent conditions.

(i) There is an invariant probability measure for $\xi$ (that is, (4.1) admits a positive solution with $\sum_{n \in \mathbb{Z}, 1 \leq j \leq m} \pi_{n}(j)=1$ );

(ii) The expected return time to each site is finite.

THEOREM 6.1. $\xi(t)$ is positive recurrent iff

$$
\sum_{n=1}^{\infty}\left\|\alpha_{1}^{-} \ldots \alpha_{n}^{-}\right\|<\infty \quad \text { and } \quad \sum_{n=1}^{\infty}\left\|\alpha_{-1} \ldots \alpha_{-n}\right\|<\infty
$$

Proof. (I) Suppose that $\xi$ is positive recurrent and let $\pi=\left(\pi_{n},-\infty<\right.$ $n<\infty$, ) be the corresponding invariant measure. We claim that then

$$
\pi_{n} P_{n} \mathbf{1}=\pi_{n+1} Q_{n} \mathbf{1} .
$$

Denote $\mathcal{P}_{\pi}(\cdot)$ the distribution of the walk $\xi$ with initial distribution $\pi$. Let $\mathfrak{p}(n, t)=\mathcal{P}_{\pi}\left(X_{t} \leq n\right)$. Since $\pi$ is invariant, $\mathfrak{p}(n, t)$ does not depend on $t$. Thus

$$
0=\mathfrak{p}(n, t+1)-\mathfrak{p}(n, t)=\pi_{n+1} Q_{n} \mathbf{1}-\pi_{n} P_{n} \mathbf{1}
$$

proving (6.2). Hence, by (4.12), $\mathfrak{c}=0$ and by Theorem 4.12(i) we have $\pi_{n}=\pi_{n-1} \alpha_{n}^{-}$. Iterating we obtain

$$
\pi_{n}=\pi_{0} \alpha_{1}^{-} \ldots \alpha_{n}^{-}
$$

Since $\pi_{n} \in l^{1}$ we must have

$$
\sum_{n=1}^{\infty}\left\|\alpha_{1}^{-} \ldots \alpha_{n}^{-}\right\|<\infty
$$

imsart-aop ver. 2014/10/16 file: InvarMesRWRE.tex date: September 18, 2018 
Likewise

$$
\sum_{n=1}^{\infty}\left\|\alpha_{-1} \ldots \alpha_{-n}\right\|<\infty
$$

(II) Let $\pi$ be the unique positive solution to (4.1) with $\mathfrak{c}=0$. Then it satisfies (6.3) as well as $\pi_{-n}=\pi_{0} \alpha_{-1} \ldots \alpha_{-n}$. Hence if (6.1) holds then $\pi_{n} \in l^{1}(\mathbb{S})$ and $\xi(t)$ is positive recurrent.

It is a standard fact that the walk on a strip is positive recurrent iff its restrictions to both positive and negative semi-strips are positive recurrent. Therefore we obtain the following result.

COROLlary 6.2. (a) The restriction of $\xi(t)$ to the positive semistrip is positive recurrent iff

$$
\sum_{n=1}^{\infty}\left\|\alpha_{1}^{-} \ldots \alpha_{n}^{-}\right\|<\infty
$$

(b) The restriction of $\xi(t)$ to the negative semistrip is positive recurrent iff

$$
\sum_{n=1}^{\infty}\left\|\alpha_{-1} \ldots \alpha_{-n}\right\|<\infty
$$

Proof. We prove (a), the proof of (b) is similar.

Consider a modified MC, where the transition probabilities for $n \geq 0$ are the same as for the original walk, and for $n<0$ the modified transition probabilities $\tilde{\mathcal{Q}}_{n}$ do not depend on $n$ and have a drift to the right. Then for the modified walk $\sum_{n=1}^{\infty}\left\|\tilde{\alpha}_{-1} \ldots \tilde{\alpha}_{-n}\right\|<\infty$. Hence the positive recurrence is equivalent to the convergence of $\sum_{n=1}^{\infty}\left\|\alpha_{1}^{-} \ldots \alpha_{n}^{-}\right\|<\infty$. On the other hand since the restriction of the modified walk to the negative semistrip is obviously positive recurrent, the positive recurrence on the strip is equivalent to the positive recurrence of the restriction of our original walk on the positive semistrip.

Corollary 6.3. Suppose that an environment $\omega=\left\{\left(P_{n}, Q_{n}, R_{n}\right)\right\}_{n \in \mathbb{Z}_{+}}$ satisfies (2.6) then the restriction of the walk on the positive semistrip is null recurrent iff

$$
\sum_{n=1}^{\infty}\left(\left\|\alpha_{1}^{-} \ldots \alpha_{n}^{-}\right\|\right)^{-1}=\infty \quad \text { and } \quad \sum_{n=1}^{\infty}\left\|\alpha_{1}^{-} \ldots \alpha_{n}^{-}\right\|=\infty
$$

imsart-aop ver. 2014/10/16 file: InvarMesRWRE.tex date: September 18, 2018 
Proof. Combining Theorem 3.5, Remark 3.3 and Corollary 6.2 we see that null recurrence is equivalent to

$$
\sum_{n=1}^{\infty}\left\|\alpha_{n} \ldots \alpha_{1}\right\|=\infty \quad \text { and } \quad \sum_{n=1}^{\infty}\left\|\alpha_{1}^{-} \ldots \alpha_{n}^{-}\right\|=\infty
$$

It remains to observe that the product $\left\|\alpha_{n} \ldots \alpha_{1}\right\|\left\|\alpha_{1}^{-} \ldots \alpha_{n}^{-}\right\|$is uniformly bounded from above and below, see [3, equation (3.19)] (note that [3] uses the notations $\hat{\alpha}_{n}, \hat{\beta}_{n}$ instead of our $\alpha_{n}$ and $\alpha_{n}^{-}$).

For strips of width 1, Remark 3.2 shows that the null recurrence condition is equivalent to the divergence of both

$$
\sum_{n=1}^{\infty} \prod_{j=1}^{n}\left(\frac{q_{j}}{p_{j}}\right) \quad \text { and } \quad \sum_{n=1}^{\infty} \prod_{j=1}^{n}\left(\frac{p_{j}}{q_{j}}\right) .
$$

Namely, the divergence of the first series is equivalent to recurrence and the convergence ot the second series is equivalent to positive recurrence. Thus we recover the well known criterion for null recurrence of birth and death chains (see [13], Sections 6.4 and 6.5).

COROLlARY 6.4. In a stationary ergodic environment, the restriction of the walk to the positive semistrip is positive recurrent iff $\boldsymbol{\lambda}^{+}>0$.

We omit the derivation of Corollary 6.4 from Corollary 6.2 as it is the same as the derivation of Theorem 3.6 from Theorem 3.5.

6.2. Answers to some questions from [33]. The following model generalizes the one studied by Ya. Sinai in [33]. Let (as in Introduction) $T$ be a measure preserving ergodic automorphism acting on a measure space $(M, \mathcal{M}, \mu)$. (Here and throughout this section the notations are as in [33]. In particular elements of $M$ are denoted by $x$.)

Suppose that we are given a function on $M$ taking values in $\mathcal{J}$ (see (2.4)): $x \mapsto(P(x), Q(x), R(x))$. Define a random walk on $\bar{M} \stackrel{\text { def }}{=} M \times[1, \ldots m]$ as follows: if at time $t$ the particle is at $(x, i) \in \bar{M}$ then at time $t+1$ it jumps to $(T x, j)$ with probability $P(x, i, j)$, to $\left(T^{-1} x, j\right)$ with probability $Q(x, i, j)$, and to $(x, j)$ with probability $R(x, i, j)$.

To transform this RW into a RW on the strip $\mathbb{S}=\mathbb{Z} \times[1, \ldots m]$ we set

$$
\left(P_{0}(x), Q_{0}(x), R_{0}(x)\right)=(P(x), Q(x), R(x))
$$


(and thus $\left.\left(P_{n}(x), Q_{n}(x), R_{n}(x)\right)=\left(P\left(T^{n} x\right), Q\left(T^{n} x\right), R\left(T^{n} x\right)\right)\right)$. The RW starting from $(x, i) \in \bar{M}$ is thus mapped into a walk starting from $(0, i)$. The ergodicity condition is automatically satisfied but in addition we have to impose on our matrices the ellipticity condition (2.6).

After that, all the results about the existence of the invariant density on the space of environments on the strip are automatically translated into the statements about the invariant density on $\bar{M}$.

The above construction also answers the more general question from [33]. We state it now in a form which is slightly more precise than in the Introduction. Consider a random walk on $M$ where a particle can jump from $x \in M$ to $T^{i} x,(|i| \leq m)$ with probability $p(x, i), \sum_{i=-m}^{m} p(x, i)=1$. This walk transforms into a walk on $\mathbb{R}$ with jumps of length $\leq m$ in exactly the same way as above. As we have already mentioned before, the latter walk is reduced to a walk on a strip of width $m$ and this answers the question.

Let us return to the model considered in $[33,17,18]$. This model is obtained from the above model on $\bar{M}$ by setting $m=1$.

As in [33], we shall consider two cases.

6.2.1. The symmetric (recurrent) case. In [33] the RW on $M$ is called symmetric if

$$
\int_{M} \ln \frac{1-p(x)}{p(x)} \mu(d x)=0 .
$$

Since $\boldsymbol{\lambda}^{+}=0$ (as it is equal to this integral), Theorem 3.6 implies that the corresponding random walk on $\mathbb{Z}$ is recurrent. Recall that, according to Remark 3.2, $\tilde{\lambda}(x)=\frac{q(T x)}{p(x)}$. Theorem 5.5 implies the following statement.

Corollary 6.5. Consider the Markov chain on $M$ with $p$ satisfying strong ellipticity condition: there is $\varepsilon>0$ such that $\varepsilon \leq p \leq 1-\varepsilon$. Suppose also that $\boldsymbol{\lambda}^{+}=0$. Then the invariant measure of the Markov chain on $M$ has a density with respect to $\mu$ if and only if there exists a non-negative function $\tilde{\beta}: M \mapsto \mathbb{R}$ such that

$$
\frac{q(T x)}{p(x)}=\frac{\tilde{\beta}(x)}{\tilde{\beta}(T x)} \text { for } \mu \text {-a.a. } x \in M \text { and } \int_{M} \tilde{\beta}(x) \mu(d x)<\infty .
$$

REMARK 6.6. The homological equation considered in [33, equation (2)] reads $\frac{p(x)}{1-p(x)}=\frac{h(x)}{h\left(T^{-1} x\right)}$. This equation and (6.4) are equivalent in the sense that whenever one of them has a solution so does the other. A more general equivalence of (5.6) and the equation $\lambda(\omega)=\frac{\beta(T \omega)}{\beta(\omega)}$ (where $\lambda(\omega)$ is as in (3.14)) was shown in [11, Lemma 3.2].

imsart-aop ver. 2014/10/16 file: InvarMesRWRE.tex date: September 18, 2018 
6.2.2. The non-symmetric (transient) case. In [33] the RW on $M$ is said to be non-symmetric if

$$
\int_{M} \ln \frac{p(x)}{1-p(x)} \mu(d x)>0 .
$$

This inequality implies that $\boldsymbol{\lambda}^{+}<0$ and by Theorem 3.6 the corresponding walk on $\mathbb{Z}$ is transient to the right. By Theorem 5.4 the invariant density now exists if and only if

$$
\int_{M} \frac{1}{p(x)}\left(\sum_{n=0}^{\infty} \prod_{k=1}^{n} \frac{1-p\left(T^{k} x\right)}{p\left(T^{k} x\right)}\right) \mu(d x)<\infty
$$

and has the form

$$
\rho(x)=\mathfrak{c} \frac{1}{p(x)}\left(\sum_{n=0}^{\infty} \prod_{k=1}^{n} \frac{1-p\left(T^{k} x\right)}{p\left(T^{k} x\right)}\right) .
$$

REMARK 6.7. The fact that the finiteness of the expectation (6.5) implies the existence of the invariant density of the form (6.6) (the if direction of this statement) was first proved in [1, Theorem 3.1] and later (and independently) in [17]. As has been shown above, the only if direction follows from the fact that the invariant density has to satisfy the invariant density equation and the only "relevant" solution it has is given by (6.6). In other words, if the expectation (6.5) is infinite then the walk on the space of environments does not have an absolutely continuous invariant measure.

6.2.3. Non-symmetric (transient) walks generated by a uniquely ergodic $T$. The random walks on a $d$-dimensional torus generated by irrational rotations (as in [33]) are a very particular case of the walks on compact metric spaces generated by uniquely ergodic automorphisms.

So suppose now that $M$ is a compact metric space and $T$ is a uniquely ergodic automorphism of $M$. We recall that $T$ is said to be uniquely ergodic if there is only one $T$-invariant measure $\mu$ on $M$. Uniquely ergodic automorphisms have the following important property: if $f: M \mapsto \mathbb{R}$ is a continuous function then

$$
\frac{1}{n} \sum_{j=0}^{n-1} f\left(T^{j} x\right) \rightarrow \int_{M} f(y) \mu(d y) \quad \text { uniformly in } x \in M .
$$

Consider again the RW on $\bar{M}$ and suppose that the triple $(P(x), Q(x), R(x))$ is continuous in $x$ and satisfies the ellipticity conditions (2.6). Then also the

imsart-aop ver. 2014/10/16 file: InvarMesRWRE.tex date: September 18, 2018 
function $\tilde{\lambda}(x)$ (see (3.14)) is continuous in $x$. We suppose now that the corresponding walk on the strip is transient to the right:

$$
\boldsymbol{\lambda}^{+}=\int_{M} \ln \tilde{\lambda}(x) \mu(d x)<0 .
$$

Now, due to (3.12) and to unique ergodicity, the following sequence converges uniformly in $x$ and $n$ :

$$
\frac{1}{k} \ln \left\|l_{n+k} \alpha_{n+k-1} \ldots \alpha_{n}\right\|=\frac{1}{k} \sum_{i=0}^{k-1} \ln \tilde{\lambda}_{n+i} \rightarrow \boldsymbol{\lambda}^{+} \text {as } k \rightarrow \infty .
$$

This uniform convergence together with Remark 3.3 imply that also the series in (5.1) converges uniformly in $x$ and hence $\boldsymbol{\rho}(x)$ is a continuous function of $x$ and in particular is integrable. We thus proved that in the asymmetric (transient) case the $M C$ on $\bar{M}$ generated by a uniquely ergodic automorphism $T$ always has an invariant density. Moreover, this density is continuous.

6.3. The Green Function. Consider the case where the random walk escapes to the right, that is, $X_{t} \rightarrow+\infty$ as $t \rightarrow \infty$. In this case each site is visited only finitely many times with probability one and one can consider the Green function $g((l, i) ;(n, j))$ which is the expected number of visits to the site $(n, j)$ given that the walk starts from $(l, i)$.

Lemma 6.8. The following limit exists and does not depend on $i$

$$
\mathfrak{g}_{n}(j)=\lim _{l \rightarrow-\infty} g((l, i),(n, j)) .
$$

Proof. Let $\sigma_{n}$ be the time of the first visit by the walker to level n. (If the walker starts from $(l, i)$ with $l<n$ then $\sigma_{n}$ is finite with probability one since the walk escapes to the right). Then

$$
g((l, i) ;(n, j))=\sum_{k=1}^{m} \mathcal{P}\left(\xi\left(\sigma_{n}\right)=(n, k) \mid \xi(0)=(l, i)\right) g((n, k) ;(n, j)) .
$$

Since the second factor in the last expression does not depend on $l$ and the first one converges as $l \rightarrow-\infty$ to $y_{n}(k)$ where $y_{n}$ is given by (3.5), the result follows.

Lemma 6.9. Let $\mathfrak{g}_{n}$ be the vector with components $\mathfrak{g}_{n}(i)$. Then $\mathfrak{g}_{n}$ is a monotone solution to (2.9) and the corresponding value of $\mathfrak{c}=1$.

imsart-aop ver. 2014/10/16 file: InvarMesRWRE.tex date: September 18, 2018 
Proof. Let $\tau_{k}$ be the time of the $k^{\text {th }}$ visit to the set $L_{n} \cup L_{n+1}$ (see the definitions in section 2.1). Then $\bar{\xi}_{k}:=\xi\left(\tau_{k}\right)$ is a Markov chain. Let

$$
\begin{aligned}
& U_{n}(i, j):=\mathcal{P}\left(\bar{\xi}_{k+1}=(n, j) \mid \bar{\xi}_{k}=(n, i)\right)=R_{n}(i, j)+\sum_{s=1}^{m} Q_{n}(i, s) \zeta_{n-1}(s, j), \\
& (6.8) \quad V_{n}(i, j):=\mathcal{P}\left(\bar{\xi}_{k+1}=(n, j) \mid \bar{\xi}_{k}=(n+1, i)\right)=Q_{n+1}(i, j) .
\end{aligned}
$$

Thus

$$
g_{n}((l, s) ;(n, j))=\mathcal{P}\left(\xi\left(\sigma_{n}\right)=(n, j) \mid \xi(0)=(l, s)\right)+\sum_{k=1}^{\infty} \mathcal{P}\left(\bar{\xi}_{k+1}=(n, j) \mid \xi(0)=(l, s)\right) .
$$

Using (6.7) and (6.8) we can rewrite the last sum as

$$
\sum_{k=1}^{\infty} \sum_{i=1}^{m}\left[\mathcal{P}\left(\bar{\xi}_{k}=(n, i) \mid \xi(0)=(l, s)\right) U_{n}(i, j)+\mathcal{P}\left(\bar{\xi}_{k}=(n+1, i) \mid \xi(0)=(l, s)\right) V_{n}(i, j)\right] .
$$

Therefore

$$
\begin{aligned}
g((n, j) ;(l, s)) & =\mathcal{P}\left(\xi\left(\sigma_{n}\right)=(n, j) \mid \xi(0)=(l, s)\right) \\
& +\sum_{i=1}^{m}\left[g((n, i) ;(l, s)) U_{n}(i, j)+g((n+1, i) ;(l, s)) V_{n}(i, j)\right] .
\end{aligned}
$$

Letting $l \rightarrow-\infty$ we obtain

$$
\mathfrak{g}_{n}(j)=y_{n}(j)+\sum_{i=1}^{m}\left[\mathfrak{g}_{n}(i) U_{n}(i, j)+\mathfrak{g}_{n+1}(j) V_{n}(i, j)\right] .
$$

Remembering (6.7) and (6.8) we obtain

$$
\mathfrak{g}_{n}=\mathfrak{g}_{n} R_{n}+\mathfrak{g}_{n} Q_{n} \zeta_{n-1}+\mathfrak{g}_{n+1} Q_{n+1}+y_{n} .
$$

Hence

$$
\mathfrak{g}_{n}\left(I-R_{n}-Q_{n} \zeta_{n-1}\right)=\mathfrak{g}_{n+1} Q_{n+1}+y_{n} .
$$

Multiplying by $\gamma_{n}$ on the right, we obtain $\mathfrak{g}_{n}=\mathfrak{g}_{n+1} \alpha_{n}+y_{n} \gamma_{n}$. Thus letting $\mathfrak{h}_{n}=\mathfrak{g}_{n}-\mathfrak{g}_{n+1} \alpha_{n}$ we obtain $\mathfrak{h}_{n}=y_{n} \gamma_{n}$. Now Lemma 4.8 gives $\mathfrak{c}=1$.

REMARK 6.10. Lemma 6.9 shows that $\mathfrak{g}_{n}$ is given by $(4.15)$ with $\mathfrak{c}=1$. The same result was derived in [10] under slightly more restrictive assumptions. Here we see that this result follows immediately from the theory developed in this paper.

imsart-aop ver. 2014/10/16 file: InvarMesRWRE.tex date: September 18, 2018 
REMARK 6.11. If the environment is stationary and the random walk escapes to the right, (that is, $\boldsymbol{\lambda}^{+}<0$ ) then the solution given by Lemma 6.9 is stationary by construction. In contrast, Lemma 4.4 provides another solution with $\mathfrak{c}=0$ but this solution is not stationary, since it decays exponentially by the definition of $\boldsymbol{\lambda}^{+}$.

6.4. The speed of the random walk. We shall now consider the following question: given that the RW $\xi$ (defined in (2.3)) is transient to the right, what is the speed at which it escapes to $+\infty$ ?

In this section, it will be important to use a more 'compleat' notation for the RW, namely we write $\xi_{\omega,(0, i)}(t)=\left(X_{\omega, i}(t), Y_{\omega, i}(t)\right)$ for the coordinates of the walk starting from $(0, i)$ in a given environment $\omega$ and evaluated at time $t$. We recall the natural definition of the speed: $\mathrm{v}=\lim _{t \rightarrow \infty} \frac{X_{\omega, i}(t)}{t}$ if this limit exists.

In the case of the RWRE on a strip, the answer to this question was found by a different method in [15] and independently in [30]. Here, we shall show how one can compute the speed in terms of the invariant density.

Theorem 6.12. Suppose that the Markov chain $\left(\bar{\omega}_{t}, Y_{t}\right)$ defined by (2.7) has the invariant density $\boldsymbol{\rho}$. Then for any initial point $(0, i)$, for $\mathbb{P}$-a.a. $\omega$, and for $\mathcal{P}_{\omega,(0, i)}$-a.a. trajectories $\xi_{\omega, i}(\cdot)$ the following limit exists:

$$
\mathrm{v}=\lim _{t \rightarrow \infty} \frac{X_{\omega, i}(t)}{t}=\mathfrak{c}, \text { where } \mathfrak{c} \text { is the constant defined in }(5.5) .
$$

Proof. We shall use the following fact.

Lemma 6.13. If the Markov chain $\left(\bar{\omega}_{t}, Y_{t}\right)$ has the invariant density $\rho$ then for $\mathbb{P}$-a.a. $\omega$ and all starting points $(0, i)$ the speed $\mathrm{v}$

$$
\mathrm{v}=\lim _{t \rightarrow \infty} \frac{X_{\omega, i}(t)}{t}=\mathbb{E}_{\rho}(X(1)) \text { with } \mathcal{P}_{\omega,(0, i)} \text {-probability } 1,
$$

where $\mathbb{E}_{\rho}$ is the expectation with respect to the measure $\rho(\omega, i) \mathbb{P}(d \omega) \mathcal{P}_{\omega,(0, i)}(d \xi)$ and $X(1)$ is the first coordinate of $\xi(1)$.

For a beautiful proof of this Lemma we refer the reader to [5], page 14 . The proof presented there is explained in a somewhat different setting but the adjustments needed for our case are routine.

So, for us it remains to compute the expectation. To this end, note first that

$$
\mathrm{E}_{\omega,(0, i)}\left(X_{\omega,(0, i)}(1)\right)=\sum_{j=1}^{m}\left(P_{0}(i, j)-Q_{0}(i, j)\right)
$$

imsart-aop ver. 2014/10/16 file: InvarMesRWRE.tex date: September 18, 2018 
and hence

$$
\begin{aligned}
\mathbb{E}_{\rho}(X(1)) & =\mathbb{E}\left(\sum_{i=1}^{m} \rho(\omega, i) \mathrm{E}_{\omega,(0, i)}(X(1))\right) \\
& =\mathbb{E}\left(\sum_{i=1}^{m} \rho(\omega, i) \sum_{j=1}^{m}\left(P_{0}(i, j)-Q_{0}(i, j)\right)\right) \\
& =\mathbb{E}\left(\boldsymbol{\rho}\left(P_{0}-Q_{0}\right) \mathbf{1}\right)=\mathbb{E}\left(\boldsymbol{\rho} P_{0} \mathbf{1}\right)-\mathbb{E}\left(\boldsymbol{\rho} Q_{0} \mathbf{1}\right) .
\end{aligned}
$$

Since $\mathbb{E}\left(\boldsymbol{\rho} Q_{0} \mathbf{1}\right)=\mathbb{E}\left(\boldsymbol{\rho}(T \omega) Q_{0}(T \omega) \mathbf{1}\right)=\mathbb{E}\left(\boldsymbol{\rho}_{1} Q_{1} \mathbf{1}\right)$ we have

$$
\mathrm{v}=\mathbb{E}\left(\boldsymbol{\rho} P_{0} \mathbf{1}-\boldsymbol{\rho}_{1} Q_{1} \mathbf{1}\right)=\mathfrak{c}
$$

because $\boldsymbol{\rho} P_{0} \mathbf{1}-\boldsymbol{\rho}_{1} Q_{1} \mathbf{1}=\mathfrak{c}$ by (4.12).

\section{APPENDIX A: DERIVATION OF THE INVARIANT DENSITY EQUATION.}

Proof of Lemma 2.3.. By the definition of the invariant measure we have that for any continuous function $f: \bar{\Omega} \mapsto \mathbb{R}$ the following equality holds:

$$
\begin{aligned}
& \sum_{j=1}^{m} \int_{\Omega} f(\omega, j) \rho(\omega, j) \mathbb{P}(d \omega)=\sum_{i=1}^{m} \int_{\Omega}(K f)(\omega, i) \rho(\omega, i) \mathbb{P}(d \omega) \\
& =\sum_{i=1}^{m} \int_{\Omega}\left(\sum_{k \in\{-1,0,1\}, 1 \leq j \leq m} \mathcal{Q}_{\omega}((0, i),(k, j)) f\left(T^{k} \omega, j\right)\right) \rho(\omega, i) \mathbb{P}(d \omega) .
\end{aligned}
$$

Rearranging the sums and taking into account that $T$ is preserving $\mathbb{P}$, we can present the right hand side of (A.1) as

$$
\begin{aligned}
& \sum_{j=1}^{m} \sum_{i=1}^{m} \sum_{k \in\{-1,0,1\}} \int_{\Omega} \mathcal{Q}_{\omega}((0, i),(k, j)) f\left(T^{k} \omega, j\right) \rho(\omega, i) \mathbb{P}(d \omega)= \\
& \sum_{j=1}^{m} \sum_{i=1}^{m} \sum_{k \in\{-1,0,1\}} \int_{\Omega} \mathcal{Q}_{T^{-k} \omega}((0, i),(k, j)) f(\omega, j) \rho\left(T^{-k} \omega, i\right) \mathbb{P}(d \omega)= \\
& \sum_{j=1}^{m} \int_{\Omega}\left(\sum_{k \in\{-1,0,1\}} \sum_{i=1}^{m} \rho\left(T^{-k} \omega, i\right) \mathcal{Q}_{T^{-k}}((0, i),(k, j))\right) f(\omega, j) \mathbb{P}(d \omega) .
\end{aligned}
$$

imsart-aop ver. 2014/10/16 file: InvarMesRWRE.tex date: September 18, 2018 
Since $f(\omega, j)$ are arbitrary continuous functions, it follows that for $\mathbb{P}$-almost all $\omega$

$$
\rho(\omega, j)=\sum_{k \in\{-1,0,1\}} \sum_{i=1}^{m} \rho\left(T^{-k} \omega, i\right) \mathcal{Q}_{T^{-k} \omega}((0, i),(k, j))
$$

By $(2.2)$ we have $\mathcal{Q}_{T^{-1} \omega}((0, i),(1, j))=P_{-1}(i, j), \mathcal{Q}_{\omega}((0, i),(0, j))=R_{0}(i, j)$, and $\mathcal{Q}_{T \omega}((0, i),(-1, j))=Q_{1}(i, j)$ and thus

$$
\rho(\omega, j)=\sum_{i=1}^{m} \rho(T \omega, i) Q_{1}(i, j)+\sum_{i=1}^{m} \rho(\omega, i) R_{0}(i, j)+\sum_{i=1}^{m} \rho\left(T^{-1} \omega, i\right) P_{-1}(i, j) .
$$

It remains to notice that the last equation can be re-written in the vector form as

$$
\boldsymbol{\rho}(\omega)=\boldsymbol{\rho}(T \omega) Q_{1}+\boldsymbol{\rho}(\omega) R_{0}+\boldsymbol{\rho}\left(T^{-1} \omega\right) P_{-1} .
$$

Finally, replacing in this equation $\omega$ by $T^{n} \omega$ we obtain (2.9).

We thus proved that (A.1) implies (A.2) and (2.9).

Suppose now that we are given a sequence $\boldsymbol{\rho}_{n}(\omega)$ which satisfies $(2.9)$, (2.10), and (2.11). This in particular means that (A.2) holds true and we obtain (A.1) by reversing each step in the above proof.

\section{APPENDIX B: RECURRENCE OF GENERAL WALKS.}

Here we prove Theorem 3.5.

Proof. It suffices to prove part (a), the proof of part (b) is similar.

Given $a, b \in \mathbb{Z}$ and $n \in[a, b]$, let $\mathfrak{l}_{n}$ be the vector with components

$$
\mathfrak{l}_{n}(j)=\mathcal{P}\left(\xi \text { visits level b before } a \mid \xi_{0}=(n, j)\right) .
$$

It is proven in [3] that

$$
\mathfrak{l}_{n}=\varphi_{n} \varphi_{n+1} \ldots \varphi_{b-1} \mathbf{1}
$$

where $\varphi_{n}$ is defined recursively by

$$
\varphi_{n}=\left(I-R_{n}-Q_{n} \varphi_{n-1}\right)^{-1} P_{n} \text { if } n \geq a+1 \text { starting with } \varphi_{a}=0 .
$$

(I) Suppose that series (3.24) converges. Set $\Delta_{n}=\zeta_{n}-\varphi_{n}$, where $\zeta_{n}$ is as in (3.2). Then $\varphi_{n}=\zeta_{n}-\Delta_{n}$ and

$$
\begin{aligned}
\mathfrak{l}_{n} & =\left(\zeta_{n}-\Delta_{n}\right)\left(\zeta_{n+1}-\Delta_{n+1}\right) \ldots\left(\zeta_{b-1}-\Delta_{b-1}\right) \mathbf{1} \\
& \geq\left(1-\left\|\Delta_{n}\right\|\right)\left(1-\left\|\Delta_{n+1}\right\|\right) \ldots\left(1-\left\|\Delta_{b-1}\right\|\right) \mathbf{1} .
\end{aligned}
$$

imsart-aop ver. 2014/10/16 file: InvarMesRWRE.tex date: September 18, 2018 
Since

$$
\begin{aligned}
\Delta_{n} & =\left[\left(I-R_{n}-Q_{n} \zeta_{n-1}\right)^{-1}-\left(I-R_{n}-Q_{n} \varphi_{n-1}\right)^{-1}\right] P_{n} \\
& =\left(I-R_{n}-Q_{n} \zeta_{n-1}\right)^{-1} Q_{n}\left(\zeta_{n-1}-\varphi_{n-1}\right)\left(I-R_{n}-Q_{n} \varphi_{n-1}\right)^{-1} P_{n}
\end{aligned}
$$

we see that $\Delta_{n}=A_{n} \Delta_{n-1} \varphi_{n-1}$ and hence

$$
\Delta_{n}=A_{n} A_{n-1} \ldots A_{a+1} \Delta_{a} \varphi_{a+1} \ldots \varphi_{n} .
$$

Thus, for $a=0$ we get $\left\|\Delta_{n}\right\| \leq\left\|A_{n} \ldots A_{1}\right\|$ and so if (3.24) converges then (B.1) implies that there is a constant $\kappa>0$ such that $\mathfrak{l}_{n} \geq \kappa \mathbf{1}$ uniformly in $b$. Taking $b \rightarrow+\infty$ we obtain that $\mathcal{P}\left(X_{t} \rightarrow+\infty\right)>0$.

(II) Suppose now that $\mathcal{P}\left(X_{t} \rightarrow \infty\right)>0$. It follows that $\mathfrak{l}_{0}>\kappa \mathbf{1}$ for some $\kappa>0$. We need the following

LEMMA B.1. There is a constant $\hat{\varepsilon}>0$ such that

$$
\Delta_{n} \mathbf{1} \geq \hat{\varepsilon}\left\|\Delta_{n}\right\| \mathbf{1}
$$

Proof. Since $\Delta_{n} \mathbf{1}=A_{n} \Delta_{n-1} \varphi_{n-1} \mathbf{1}=A_{n} u$ with $u=\Delta_{n-1} \varphi_{n-1} \mathbf{1}>0$, we have

$$
\frac{\sum_{j=1}^{m} \Delta_{n}(i, j)}{\sum_{j=1}^{m} \Delta_{n}(k, j)}=\frac{\sum_{j=1}^{m} A_{n}(i, j) u_{j}}{\sum_{j=1}^{m} A_{n}(k, j) u_{j}} \geq \min _{j} \frac{A_{n}(i, j)}{A_{n}(k, j)} \geq \frac{\varepsilon}{\text { Const }},
$$

where the last inequality is due to (3.13). Hence

$$
\frac{\sum_{j=1}^{m} \Delta_{n}(i, j)}{\left\|\Delta_{n}\right\|}=\frac{\sum_{j=1}^{m} \Delta_{n}(i, j)}{\max _{k} \sum_{j} \Delta_{n}(k, j)} \geq \frac{\varepsilon}{\text { Const }} \equiv \hat{\varepsilon} .
$$

Thus $\left(\zeta_{n}-\Delta_{n}\right) \mathbf{1}=\mathbf{1}-\Delta_{n} \mathbf{1} \leq\left(1-\hat{\varepsilon}\left\|\Delta_{n}\right\|\right) \mathbf{1}$ and we obtain by induction that

$$
\mathfrak{l}_{0}=\left(\zeta_{0}-\Delta_{0}\right) \ldots\left(\zeta_{b-1}-\Delta_{b-1}\right) \mathbf{1} \leq\left(1-\hat{\varepsilon}\left\|\Delta_{0}\right\|\right) \ldots\left(1-\hat{\varepsilon}\left\|\Delta_{b-1}\right\|\right) \mathbf{1} .
$$

Hence

$$
\prod_{n=0}^{\infty}\left(1-\hat{\varepsilon}\left\|\Delta_{n}\right\|\right) \geq \kappa
$$

and therefore

$$
\sum_{n=0}^{\infty}\left\|\Delta_{n}\right\|<\infty
$$

imsart-aop ver. 2014/10/16 file: InvarMesRWRE.tex date: September 18, 2018 
On the other hand (B.2) implies

$$
\left\|\Delta_{n}\right\|=\left\|\Delta_{n} \mathbf{1}\right\| \geq\left\|A_{n} \ldots A_{1} \mathbf{1}\right\| \prod_{k=1}^{n}\left(1-\left\|\Delta_{k}\right\|\right)=c\left\|A_{n} \ldots A_{1}\right\|,
$$

where $c=\prod_{k=1}^{\infty}\left(1-\left\|\Delta_{k}\right\|\right)>0$ because of (B.3). From the last two displays we obtain that (3.24) converges as needed.

\section{REFERENCES}

[1] S.Alili: Asymptotic behaviour for random walks in random environments. J. Appl. Prob. 36 (1999) 334-349 .

[2] N. Berger, M. Cohen, and R. Rosenthal: Local limit theorem and equivalence of dynamic and static points of view for certain ballistic random walks in i.i.d. environments. The Annals of Probability 44 No. 4 (2016), 2889-2979.

[3] E. Bolthausen, I. Goldsheid: Recurrence and transience of random walks in random environments on a strip. Commun. Math. Phys. 214 (2000), 429-447 .

[4] E. Bolthausen, I. Goldsheid: Lingering random walks in random environment on a strip. Comm. Math. Phys. 278, No. 1 (2008), 253-288.

[5] E. Bolthausen, A.-S. Sznitman: Ten lectures on random media. DMV Seminar, 32 Birkhauser, Basel (2002).

[6] E. Bolthausen, A.-S. Sznitman: On the static and dynamic poins of view for certain random walks in random environment. Methods and Applications of Analysis 9, No. 3 (2002), 345-376.

[7] J. Brémont: One-dimensional finite range random walk in random medium and invariant measure equation. Ann. Inst. H. Poincare Prob/Stat, 45 (2009) 70-103.

[8] J.-D. Deuschel, X. Guoy, and A. F. Ramírez: Quenched invariance principle for random walk in time-dependent balanced random environment. (4 Sept. 2016) Avalable at https://arxiv.org/pdf/1503.01964.pdf.

[9] D. Dolgopyat and I. Goldsheid: Quenched limit theorems for nearest neighbour random walks in $1 D$ random environment. Comm. Math. Phys. 315 (2012), 241-277.

[10] D. Dolgopyat, I. Goldsheid: Limit theorems for random walks on a strip in subdiffusive regimes. Nonlinearity 26 (2013) 1743-1782.

[11] D. Dolgopyat, I. Goldsheid: The Central Limit Theorem for recurrent random walks on a strip with bounded potential. Nonlinearity 31 (2018) 3381-3412.

[12] D. Dolgopyat, I. Goldsheid: Constructive approach to limit theorems for recurrent diffusive random walks on a strip. submitted.

[13] R. Durrett: Probability: theory and examples. 4th edition. Cambridge Univ. Press, Cambridge (2010).

[14] N. Enriquez, C. Sabot, L. Tournier, O. Zindy: Quenched limits for the fluctuations of transient random walks in random environment on $\mathbb{Z}^{1}$. Ann. Appl. Probab. 23 (2013) 1148-1187.

[15] I. Goldsheid: Linear and sub-linear growth and the CLT for hitting times of a random walk in random environment on a strip. Prob. Th. Rel. Fields 141 (2008) 471-511.

[16] W. Hong, M. Zhang: Branching structure for the transient random walk on a strip in a random environment. arXiv:1204.1104v1 (2012)

imsart-aop ver. 2014/10/16 file: InvarMesRWRE.tex date: September 18, 2018 
[17] V. Kaloshin, Ya. Sinai: Nonsymmetric Random Walks along orbits of Ergodic Automorphisms. Publ. Amer. Math. Soc. Transl. Ser. 2, Vol. 198 (2000) 8 pp.

[18] V. Kaloshin, Ya. Sinai: Simple Random Walks along Orbits of Anosov Diffeomorphisms. Proc. Steklov Inst. Math., 228 (2000) 224-233

[19] H. Kesten: Sums of stationary sequences cannot grow slower than linearly. Proc. AMS 49 (1975) 205-211.

[20] H. Kesten, M. Kozlov, F. Spitzer: A limit law for random walk in a random environment. Compositio Math. 30 (1975) 145-168.

[21] S. Kozlov: Averaging Random Structers. Dokldy Akad. Nauk 5 (1978) 236-239. Translation in: Soviet Mathematics Doklady 19(4) (1978) 950-954.

[22] S. Kozlov: The method of averaging and walks in inhomogeneous environments. Uspekhi Mat. Nauk 40(238) (1985) 61-120 (Russian). Translation in: Russian Mathematical Surveys 40(2) (1985) 73-145.

[23] G. Lawler: Weak convergence of a random walk in a random environment. Commun. Math. Phys. 87 (1982), 81-87.

[24] L. Leskela, M. Stenlund: A local limit theorem for a transient chaotic walk in a frozen environment. Stochastic Process. Appl. 121 (2011) 2818-2838.

[25] M. Menshikov, S. Popov, A. Wade: Non-homogeneous random walks. Lyapunov function methods for near-critical stochastic systems. Cambridge Tracts in Math. 209 Cambridge University Press, Cambridge (2017) xviii+363 pp.

[26] G. Papanicolau, S. R. S. Varadhan: Diffusion with random coefficients. Statistics and Probability: essays in honor of C. R. Rao. G. Kallianpur, P.R. Krishnajah,, J.K. Gosh, edts., North Holland, Amsterdam 1982, 547- 552.

[27] J. Peterson, G. Samorodnitsky: Weak quenched limiting distributions for transient one-dimensional random walk in a random environment. Ann. Inst. Henri Poincare Probab. Stat. 49 (2013) 722-752.

[28] J. Peterson, G. Samorodnitsky: Weak weak quenched limits for the path-valued processes of hitting times and positions of a transient, one-dimensional random walk in a random environment. ALEA 9 (2012) 531-569.

[29] F. Rassoul-Agha: The point of view of the particle on the law of large numbers for random walks in a mixing random environment. The Annals of Probability 31 No. 3 (2003), 1441-1463.

[30] A. Roitershtein: Transient Random Waks on a Strip in a Random Environment. The Annals of Probability 36 No. 6 (2008), 2354-2387.

[31] C. Sabot: Random Dirichlet environment viewed from the particle in dimension $d>3$. The Annals of Probability 41 (2013) 722-743.

[32] Ya. G. Sinai: The limiting behavior of a one-dimensional random walk in a random medium. Theory Prob. Appl. 27 (1982) 256-268.

[33] Ya. G. Sinai: Simple random walks on tori. J. Statist. Phys. 94 (3/4) (1999) 695-708.

[34] O. Zeitouni: Random Walks in Random Environment. In Lectures on Probability Theory and Statistics. Lecture Notes in Math. 1837 (2004) 189-312. Springer, Berlin.

Department of Mathematics and

Institute of Physical Science \& Technology

UNIVERSITY OF MARYLAND

College Park, MD, 20742, USA

E-MAIL: dmitry@math.umd.edu
School of Mathematical Sciences QueEn MARY University of LONDON London E1 4NS, Great Britain E-MAIL: I.Goldsheid@qmul.ac.uk 